\title{
Abundance gradients in a sample of barred spiral galaxies ${ }^{\star}$
}

\author{
O. L. Dors Jr. and M. V. F. Copetti
}

\begin{abstract}
Laboratório de Análise Numérica e Astrofísica, Departamento de Matemática, Universidade Federal de Santa Maria, 97119-900 Santa Maria, RS, Brazil

e-mail: oli@lana.ccne.ufsm.br
\end{abstract}

Received 6 February 2004 / Accepted 14 March 2005

\begin{abstract}
We used photoionization models in order to reproduce the observed gradients of emission-line ratios for $\mathrm{H}$ II regions located in the normal spiral galaxy M 101 and in three barred spiral galaxies, namely NGC 1365, NGC 925, and NGC 1073. The behavior of the following nebular parameters across the disk of these galaxies was determined: temperature of the ionizing star $\left(T_{\text {eff }}\right)$, ionization parameter $(U)$, and the abundance ratios $\mathrm{O} / \mathrm{H}, \mathrm{N} / \mathrm{O}$, and $\mathrm{S} / \mathrm{O}$. Our $\mathrm{O} / \mathrm{H}$ predictions were found to be consistent with some empirical abundance determinations, but are overestimated by a factor of $0.1-0.4$ dex when compared to the direct abundance determinations. NGC 1073 seems to be overabundant in nitrogen compared to other spiral galaxies. No gradient of $\mathrm{S} / \mathrm{O}$ was found in the studied galaxies, and we derived a positive $T_{\text {eff }}$ gradient of $\Delta T_{\text {eff }} / \Delta R=(400 \pm 112) \mathrm{K} \mathrm{kpc}^{-1}$ and $U$ ranging from -3.0 to -2.3 . The $\mathrm{N} / \mathrm{O}$ vs. $\mathrm{O} / \mathrm{H}$ diagram in general is consistent with chemical evolution models that assume that the nitrogen synthesis has both a primary and a secondary component compared to oxygen. However, a very strong $\mathrm{N} / \mathrm{O}$ dependence on the $\mathrm{O} / \mathrm{H}$ at high abundance was found.
\end{abstract}

Key words. galaxies: abundances - ISM: abundances - galaxies: evolution - galaxies general - ISM: H II regions

\section{Introduction}

Abundance gradients in the disks of spiral galaxies play an important role in studies of galaxy formation and evolution, and chemical evolution models have been proposed to explain them. These models require knowledge of elemental (e.g., $\mathrm{O} / \mathrm{H}$ ) and differential (e.g., N/O, Ar/O, S/O) abundances across the galactic disk. In particular, N/O and $\mathrm{S} / \mathrm{O}$ abundance ratios can provide a check for stellar synthesis studies, especially for the functional form of the initial mass function and for stellar mass loss rates.

For decades many spectrophotometric observations of H II regions situated in spiral galaxies have been carried out, and now a large amount of emission-line intensity data is available in the literature. Unfortunately, accurate element abundances can only be derived by measuring temperaturesensitive line ratios, such as $[\mathrm{O}$ III $](\lambda 4959+\lambda 5007) / \lambda 4363$, which are weak or unobservable in H II regions with high metallicity and/or low excitation. Only in a very small number of high metallicity H II regions have direct abundance determinations been possible (e.g. Castellanos et al. 2002; Kennicutt et al. 2003; Garnett et al. 2004a); so in most of the cases, empirical calibrations between abundances and more easily measured line ratios have to be used to estimate metal abundances as, for example, the oxygen

^ Figures 4-12 are only available in electronic form at http://www . edpsciences.org indicators $R_{23}=([\mathrm{O} \mathrm{II}] \lambda 3727+[\mathrm{O}$ III $] \lambda 4949+\lambda 5007) / \mathrm{H} \beta$, $[\mathrm{N} \mathrm{II}] \lambda 6584 /[\mathrm{O}$ III $] \lambda 5007$, and $P=([\mathrm{O} \mathrm{III}] \lambda 4949+\lambda 5007) /$ $\mathrm{H} \beta / R_{23}$ suggested by Pagel et al. (1979), Alloin et al. (1979), and Pilyugin (2001a), respectively. Also, when no temperaturesensitive lines are available, the N/O ratio can be estimated using only the strong emission lines of these elements (Pagel et al. 1992). Recently, when employing the $P$-method Pilyugin et al. (2003) obtained an N/O vs. O/H diagram that agrees with the one found via direct abundance determinations for M 101. An alternative method that can be employed to derive abundances and also some other physical parameters of $\mathrm{H}$ II regions is the use of photoionization codes to model the spectra of these objects (e.g. Garnett 1989; Diaz et al. 1991; Henry et al. 1992; Henry \& Howard 1995; Morisset et al. 2002; Morisset 2004).

M 101 (NGC 5457) is the spiral galaxy with the largest number of $\mathrm{H}$ II regions in which electron temperatures have been measured; thus it has served as the prototype system for studying and comparing abundance gradients obtained by different methods. Several theoretical works have been carried out in order to model and interpret the spectra of its H II regions. In their pioneer work using self-consistent photoionization models Shields \& Searle (1978) provided an excellent fit to the observed spectra of three H II regions situated in M 101, while Evans (1986) employed theoretical photoionization models in order to determine oxygen and other heavy elements abundances along the M 101 disk. Henry \& Howard (1995), using a sequence of photoionization models, have questioned 
the break in the abundance gradient slope in M 101 derived by Zaritsky (1992), Scowen et al. (1992), and Vila-Costas \& Edmunds (1992).

Quite the opposite situation is found in most spiral galaxies, where the temperature-sensitive lines are only detected in a few objects. For barred spiral galaxies, especially, few studies have been carried out to study the differential abundance gradients, since in most of them only the $\mathrm{O} / \mathrm{H}$ abundance was estimated. In general, shallower $\mathrm{O} / \mathrm{H}$ gradients (Vila-Costas \& Edmunds 1992; Edmunds \& Roy 1993; Zaritsky et al. 1994; Martin \& Roy 1994) and stronger N/O gradients (Considère et al. 2000) have been found in barred galaxies in comparison with the ones in non-barred galaxies. As for S/O abundance, some authors have found that it does not vary with the oxygen abundance (e.g. Kennicutt et al. 2003), although some evidence of a systematic variation with $\mathrm{O} / \mathrm{H}$ has also been found (e.g. Vilchez et al. 1988).

In particular, the three barred galaxies NGC 1365, NGC 925, and NGC 1073 have no abundance gradients either reported or in conflict in the literature. For NGC 1365, Roy \& Walsh (1997) found a break in its oxygen abundance gradient, which was contested recently by Pilyugin (2003b). Alloin et al. (1981) then derived constant N/O values along the NGC 1365 disk, which is unusual for the majority of spiral galaxies and in disagreement with recent abundance determinations for this galaxy as obtained by Pilyugin et al. (2004). Moreover, in NGC 1365 the S/O gradient is not determined in any earlier work. In NGC 925 the gradient of $R_{23}$ index shows a flattening at middle galactocentric distances, which can indicate an abundance break. Finally, in the NGC 1073 galaxy the $\mathrm{N} / \mathrm{O}$ gradient is unknown to date.

The main purpose of this paper is to reproduce the radial trends shown by the spectroscopic data available for the three barred spiral galaxies NGC 1365, NGC 925, and NGC 1073 by employing photoionization models in order to constraint their $\mathrm{O} / \mathrm{H}, \mathrm{N} / \mathrm{O}$, and S/O abundance gradients. We also applied our procedure to the galaxy M 101 to test the reliability of our results by comparing our abundance predictions for this galaxy with estimates obtained by other methods. In particular, comparison of model predictions with direct abundance measurements is interesting since the discrepancy between these methods has varied through the years, and its nature is poorly known. Section 2 describes the data source utilized. The modeling procedure is described in Sect. 3, while the results are presented in Sect. 4. The discussion of the outcome and our final conclusions are given in Sects. 5 and 6, respectively.

\section{The data}

For each of the four galaxies studied in this paper we have compiled a set of observational emission-line ratios from existing data in the literature. The emission-line ratios considered are listed in Table 1. For M 101 the data were taken from the papers by Searle (1971), Smith (1975), Rayo et al. (1982), Torres-Peimbert et al. (1989), Garnett \& Kennicutt (1994), Kennicutt \& Garnett (1996), van Zee et al. (1998), and Garnett et al. (1999). The data for NGC 1365 were taken from the papers by Roy \& Walsh (1997), Pagel et al. (1979),
Table 1. Emission-line ratios considered.

\begin{tabular}{|c|c|}
\hline Symbol & Definition \\
\hline$R_{23}$ & $([\mathrm{O} \mathrm{II}] \lambda 3726+\lambda 3729+[\mathrm{O}$ III $] \lambda 4949+\lambda 5007) / \mathrm{H} \beta$ \\
\hline$[\mathrm{N}$ II $] /[\mathrm{O}$ II $]$ & {$[\mathrm{N}$ II $] \lambda 6548+\lambda 6584 /[\mathrm{O}$ II $] \lambda 3726+\lambda 3729$} \\
\hline$[\mathrm{S} \mathrm{II}] /[\mathrm{S} \mathrm{III}]$ & {$[\mathrm{S}$ II $] \lambda 6716+\lambda 6731 /[\mathrm{S} \mathrm{III}] \lambda 9069+\lambda 9532$} \\
\hline$[\mathrm{S} \mathrm{II}] /[\mathrm{O}$ II $]$ & {$[\mathrm{S}$ II $] \lambda 6716+\lambda 6731 /[\mathrm{O}$ II $] \lambda 3726+\lambda 3729$} \\
\hline$[\mathrm{O}$ III $] / \mathrm{H} \beta$ & $([\mathrm{O}$ III $] \lambda 4949+\lambda 5007) / \mathrm{H} \beta$ \\
\hline$[\mathrm{O}$ II $] /[\mathrm{O}$ III $]$ & {$[\mathrm{O}$ II $] \lambda 3726+\lambda 3729 /[\mathrm{O}$ III $] \lambda 5007$} \\
\hline$[\mathrm{N}$ II $] /[\mathrm{O}$ III $]$ & {$[\mathrm{N}$ II $] \lambda 6548+\lambda 6584 /[\mathrm{O}$ III $] \lambda 5007$} \\
\hline
\end{tabular}

and Alloin et al. (1981). For NGC 925 we employed the data obtained by Castellanos et al. (2002), van Zee et al. (1998), Martin \& Roy (1994), and Zaritsky et al. (1994). For NGC 1073 we used the data from Martin \& Roy (1994).

Since we have used observational data obtained with different instrumentations and techniques - such as imaging spectrophotometry using narrow-band interference filters, spectroscopy using optical fibres and long-slit - part of the scatter in the line ratios at a fixed galactocentric distance may be due to the non-homogeneity of the data source. However, uncertainties in intensities of the strong emission lines considered are estimated by the authors to be about $20 \%$ or less. In particular, Zaritsky et al. (1994) analyzed the uncertainties in their measurements arising from different techniques used to measure the line flux and from reduction uncertainties (extraction, sky subtraction, etc.), and found that errors in the emissionline intensities are less than 10\%. Kennicutt \& Garnett (1996) pointed out that the quality of their spectra is sufficiently high for observational errors not to have a meaningful influence on the dispersion of the data measured by them. After our comparison of measurements for a single nebula obtained by different authors we can verify that the scatter in data at a fixed galactocentric distance is not dominated by noise due to observational errors. For example, for the H335 nebula (Searle 5) in M 101 observed by Searle (1971) and Kennicutt \& Garnett (1996) and for the Hodge 8 nebula in NGC 1365 observed by Roy \& Walsh (1997) and by Pagel et al. (1979), discrepancies in the emission line ratios considered range from 0.03 to $0.1 \mathrm{dex}$, while the standard deviation of the data for all $\mathrm{H}$ II regions with similar galactocentric distances range from 0.2 to 0.7 dex.

\section{The modeling procedure}

We employed the photoionization code Cloudy/95.03 (Ferland 2002) to calculate a series of photoionization models that reproduce the variation well with the galactocentric distance $R$ of emission-line ratios listed in Table 1 . The free parameters of the models were basically (i) the $\mathrm{O}, \mathrm{N}$, and $\mathrm{S}$ abundances, (ii) the effective temperature of the ionizing star, and (iii) the ionization parameter $U$, as defined by $U=Q_{\mathrm{H}^{\circ}} / 4 \pi R_{\mathrm{s}}^{2} n c$; where $Q_{\mathrm{H}^{\circ}}$ is the number of hydrogen ionizing photons emitted per second by the ionizing $\operatorname{star}(\mathrm{s}), R_{\mathrm{s}}$ is the Strömgren radius, $n$ is the hydrogen density, and $c$ is the light speed. The emission line ratios considered have different sensitivities to the input parameters. 
The $R_{23}$ index is basically determined by the $\mathrm{O} / \mathrm{H}$ abundance, while $[\mathrm{O} \mathrm{III]}] / \mathrm{H} \beta,[\mathrm{O} \mathrm{II}] /[\mathrm{O}$ III $]$, and $[\mathrm{S} \mathrm{II}] /[\mathrm{S} \mathrm{IIII}]$ depend more on the effective temperature of the ionizing stars and on the ionization parameter (Pagel et al. 1979; Kennicutt et al. 2000; Dors \& Copetti 2003; Morisset et al. 2004). The observables used to modulate the change in $\mathrm{N} / \mathrm{O}$ and $\mathrm{S} / \mathrm{O}$ abundances were the $[\mathrm{N} \mathrm{II}] /[\mathrm{O} I I]$, [N III]/[O III] , and [S II]/[O II $]$ ratios (Henry \& Howard 1995).

In each model the spectrum of a single star of a given temperature scaled in flux according to the value of ionization parameter $U$, was taken as ionizing radiation. A grid of non-LTE line blanketing atmosphere models (taken from the website http://tlusty.gsfc.nasa.gov/) based on the TLUSTY code of Hubeny \& Lanz (1995) and recently calculated by Lanz \& Hubeny (2003) was utilized. We extracted from this database models with effective temperature and metallicities in the range $37.5 \leq T_{\text {eff }} \leq 50 \mathrm{kK}$ (with $\log g=4 \mathrm{~cm} / \mathrm{s}^{2}$ ) and $1 \leq Z / Z_{\odot} \leq 1 / 10$, respectively. The solar composition refers to Grevesse \& Sauval (1998) and corresponds to $\log (\mathrm{O} / \mathrm{H})=$ $-3.13, \log (\mathrm{N} / \mathrm{H})=-4.03, \log (\mathrm{S} / \mathrm{H})=-4.79$.

More recent solar chemical composition determinations available in the literature by Allende Prieto et al. (2001) and Holweger (2001) give $25 \%$ lower $\mathrm{O} / \mathrm{H}$ abundance. The atmosphere models with $T_{\text {eff }}=37.5,40,42.5$, and $45 \mathrm{kK}$ were interpolated in order to obtain models with $T_{\text {eff }}=39,41,41.5$, and $44 \mathrm{kK}$, since models for these temperatures are not available. In order to build realistic models, the metallicity of the nebula was matched with the closest available metallicity of the stellar atmosphere. The nebula was considered as a static sphere with filling factor $\epsilon=0.01$ and on electron density of $N_{\mathrm{e}}=200 \mathrm{~cm}^{-3}$. The abundances of the heavy metals were scaled with the oxygen abundance, with the exception of the $\mathrm{N}$ and $\mathrm{S}$ ones, which were free parameters. The presence of internal dust was considered and the grain abundances (van Hoof et al. 2001) were also linearly scaled with the oxygen abundance. To take depletion of refractory elements onto dust grains into account the abundances of the elements $\mathrm{Mg}, \mathrm{Al}, \mathrm{Ca}, \mathrm{Fe}$, $\mathrm{Ni}$, and $\mathrm{Na}$ were reduced by a factor of 10 , and $\mathrm{Si}$ by a factor of 2 (Garnett et al. 1995) relative to adopted abundances in each model.

In order to smooth the data, each galactic radius was divided in eight equally spaced segments, and for each one the mean values of observational line ratios under consideration and galactocentric distance were computed. Photoionization models were fitted to the local mean values. The $T_{\text {eff }}$ and $\log U$ parameters were chosen to be initially $40 \mathrm{kK}$ and -2.0 , respectively. As an initial guess we used the oxygen abundance derived from the gradients found by other authors, a solar $\mathrm{S} / \mathrm{O}$ ratio, and nitrogen abundance taken from the relation $\log (\mathrm{N} / \mathrm{O})=\log (0.034+120 \mathrm{O} / \mathrm{H})$ of Vila-Costas \& Edmunds (1993). The input parameters were changed iteratively following a trial and error procedure until a satisfactory solution was found, in the sense that for each line ratio the difference between the prediction and the observed value (average of the measurements around a given galactocentric distance) was less than the standard deviation of the mean of the observed ratio. In a few cases this goal was not achieved since we were unable to find models matching simultaneously all the
Table 2. Observed and predict logarithmic line ratios for NGC 5461.

\begin{tabular}{lrrr}
\hline \hline & & \multicolumn{2}{c}{ Photoionization models } \\
\cline { 3 - 4 } Line ratio & Observed & Single-star & Cluster \\
\hline$R_{23}$ & 0.78 & 0.80 & 0.77 \\
{$[\mathrm{~N} \mathrm{II}] /[\mathrm{O}$ III $]$} & -0.91 & -0.93 & -0.94 \\
{$[\mathrm{O}$ II $] /[\mathrm{O}$ III $]$} & -0.29 & -0.30 & -0.35 \\
{$[\mathrm{~S} \mathrm{II}] /[\mathrm{O}$ II $]$} & -0.89 & -0.90 & -0.85 \\
\hline
\end{tabular}

observed line ratios within the error bars, essentially because of the failure of the photoionization models to reproduce the observed [S II]/[S III] ratio.

In giant extragalactic $\mathrm{H}$ II regions (GEHRs), ionization is caused by $\mathrm{OB}$ associations composed of stars with different temperatures; however, the ionizing spectrum of an H II region depends mainly on the temperatures of the hottest stars in the ionizing cluster (Shields \& Tinsley 1976; Shields 1986). Hence, the ionizing spectrum of a single star of appropriate effective temperature, when properly scaled to generate the required luminosity, can mimic the radiation emitted by the cluster. In order to show that, we reproduced some emissionline intensities of the giant nebula NGC 5461 located in M 101 obtained by Kennicutt \& Garnett (1996) with photoionization models considering a stellar cluster as the ionizing source and compared the results with those from a single star atmosphere model (see Table 2). The stellar energy distributions obtained from the stellar population code STARBURST 99 (Leitherer et al. 1999) were used as input for the cluster model, assuming a cluster of instantaneous burst, Salpeter initial mass function $(\alpha=-2.35)$, stellar upper mass limit of $M_{\mathrm{up}}=80 M_{\odot}$, and $Z=Z_{\odot} / 5$. We found a good match with the observational data adopting $\log (\mathrm{O} / \mathrm{H})=-3.91, \log (\mathrm{N} / \mathrm{H})=-4.6, \log (\mathrm{S} / \mathrm{H})=$ $-5.30, \log U=-2.1$, and age of 2.6 Myr. Assuming the same abundances and ionization parameter we were also able to reproduce the line intensities with a single star atmosphere model of $T_{\text {eff }}=42 \mathrm{kK}$; so, for reasons of simplicity, we used atmosphere models for single stars in this paper for the shape of the ionizing radiation.

\section{Results}

Table 3 presents the parameters for the selected models used to represent the $\mathrm{H}$ II regions situated along the disk of the galaxies indicated. Column 1 lists the galactocentric distance $R$. Columns 2 and 3 list the effective temperature and metallicity, respectively, of the stellar atmosphere model adopted. Column 4 lists the ionization parameter and Cols. 5-7 list the abundance values utilized.

\section{1. $M 101$}

Figures 1 and 2 show the variation in the observational emission-line ratios of the compiled data for M 101 with galactocentric distance $R$ and our model results. An angular scale of $1^{\prime}=2.25 \mathrm{kpc}$ (Kennicutt \& Garnett 1996) was assumed. A linear behavior of $T_{\text {eff }}$ with $R$ was found, which can be 
Table 3. Model parameters.

\begin{tabular}{|c|c|c|c|c|c|c|}
\hline$R(\mathrm{kpc})$ & $T_{\text {eff }}\left(10^{4} \mathrm{~K}\right)$ & $Z / Z_{\odot}($ atmosphere $)$ & $\log U$ & $12+\log (\mathrm{O} / \mathrm{H})$ & $\log (\mathrm{N} / \mathrm{O})$ & $\log (\mathrm{S} / \mathrm{O})$ \\
\hline \multicolumn{7}{|c|}{ M 101} \\
\hline 3.00 & 3.90 & 1.00 & -2.40 & 8.94 & -0.65 & -1.40 \\
\hline 6.30 & 4.00 & 1.00 & -2.70 & 8.93 & -0.85 & -1.66 \\
\hline 10.5 & 4.25 & 1.00 & -2.50 & 8.82 & -1.10 & -1.66 \\
\hline 14.6 & 4.30 & 1.00 & -2.40 & 8.71 & -1.20 & -1.61 \\
\hline 18.2 & 4.40 & 1.00 & -2.45 & 8.56 & -1.20 & -1.61 \\
\hline 21.5 & 4.50 & 0.50 & -2.60 & 8.47 & -1.29 & -1.66 \\
\hline 26.0 & 4.75 & 0.25 & -2.65 & 8.18 & -1.29 & -1.66 \\
\hline 31.0 & 5.00 & 0.01 & -2.65 & 7.91 & -1.42 & -1.66 \\
\hline \multicolumn{7}{|c|}{ NGC 1365} \\
\hline 2.66 & 3.75 & 1.00 & -2.30 & 8.98 & -0.59 & -1.47 \\
\hline 6.02 & 3.90 & 1.00 & -2.80 & 8.91 & -0.85 & -1.61 \\
\hline 9.60 & 4.00 & 1.00 & -2.65 & 8.87 & -0.87 & -1.52 \\
\hline 13.11 & 4.10 & 1.00 & -2.70 & 8.82 & -0.94 & -1.51 \\
\hline 16.78 & 4.10 & 1.00 & -2.70 & 8.79 & -0.95 & -1.58 \\
\hline 20.31 & 4.25 & 1.00 & -2.70 & 8.76 & -1.05 & -1.48 \\
\hline 24.07 & 4.25 & 1.00 & -2.80 & 8.76 & -1.05 & -1.51 \\
\hline 27.66 & 4.25 & 1.00 & -2.70 & 8.71 & -1.05 & -1.45 \\
\hline \multicolumn{7}{|c|}{ NGC 925} \\
\hline 0.60 & 3.90 & 1.00 & -2.5 & 8.80 & -1.05 & -1.46 \\
\hline 3.05 & 4.10 & 1.00 & -2.5 & 8.68 & -1.14 & -1.45 \\
\hline 4.21 & 4.10 & 1.00 & -2.4 & 8.66 & -1.22 & -1.54 \\
\hline 5.34 & 4.10 & 1.00 & -2.5 & 8.64 & -1.36 & -1.52 \\
\hline 7.04 & 4.25 & 0.50 & -2.6 & 8.64 & -1.36 & -1.54 \\
\hline 8.77 & 4.25 & 0.50 & -2.6 & 8.64 & -1.42 & -1.54 \\
\hline 10.16 & 4.25 & 0.50 & -3.0 & 8.64 & -1.42 & -1.58 \\
\hline 12.86 & 4.25 & 0.50 & -3.0 & 8.52 & -1.37 & -1.51 \\
\hline \multicolumn{7}{|c|}{ NGC 1073} \\
\hline 2.46 & 4.10 & 0.5 & -3.00 & 8.56 & -0.90 & -1.66 \\
\hline 3.19 & 4.10 & 0.5 & -2.70 & 8.61 & -0.99 & -1.66 \\
\hline 3.77 & 4.15 & 0.5 & -2.72 & 8.56 & -0.91 & -1.66 \\
\hline 4.99 & 4.25 & 0.5 & -2.95 & 8.53 & -0.94 & -1.66 \\
\hline 5.61 & 4.30 & 0.5 & -2.60 & 8.51 & -1.00 & -1.66 \\
\hline 6.56 & 4.42 & 0.5 & -2.72 & 8.47 & -1.35 & -1.66 \\
\hline 7.23 & 4.42 & 0.5 & -2.68 & 8.47 & -1.24 & -1.66 \\
\hline 8.50 & 4.50 & 0.5 & -3.02 & 8.22 & -1.05 & -1.66 \\
\hline
\end{tabular}

represented by $T_{\text {eff }}\left(10^{4} \mathrm{~K}\right)=(0.037 \pm 0.002) R(\mathrm{kpc})+(3.77 \pm$ $0.03)$. No systematic variation of the ionization parameter along the disk was found, and we obtained a mean value of $\log U=-2.54 \pm 0.04$, in agreement with Kennicutt \& Garnett (1996), who used the correlation of $U$ with the [S II]/[S III] line ratio suggested by Díaz et al. (1991). However, Henry \& Howard (1995) and Evans (1986), using photoionization models, concluded that M 101 presents a $U$ gradient, although their gradients are slightly inconsistent, possibly due to differences in the stellar atmosphere chosen in their models.
In Fig. 3 we show our gradients for $\mathrm{O} / \mathrm{H}, \mathrm{N} / \mathrm{O}$, and $\mathrm{S} / \mathrm{O}$ and the abundances derived from direct electron temperature measurements for individual $\mathrm{H}$ II regions found in the literature (Kennicutt et al. 2003; Rayo et al. 1982; Torres-Peimbert et al. 1989; van Zee et al. 1998). Also shown in this figure are the model results of Evans (1986), who built individual photoionization models in order to reproduce the observations of the $\mathrm{H}$ II regions H 40, NGC 5461, and NGC 5471, and the O/H gradient calculated via $P$-method and via $R_{23}$ index by Pilyugin (2001b) using the Edmunds \& Pagel (1984) calibration. 


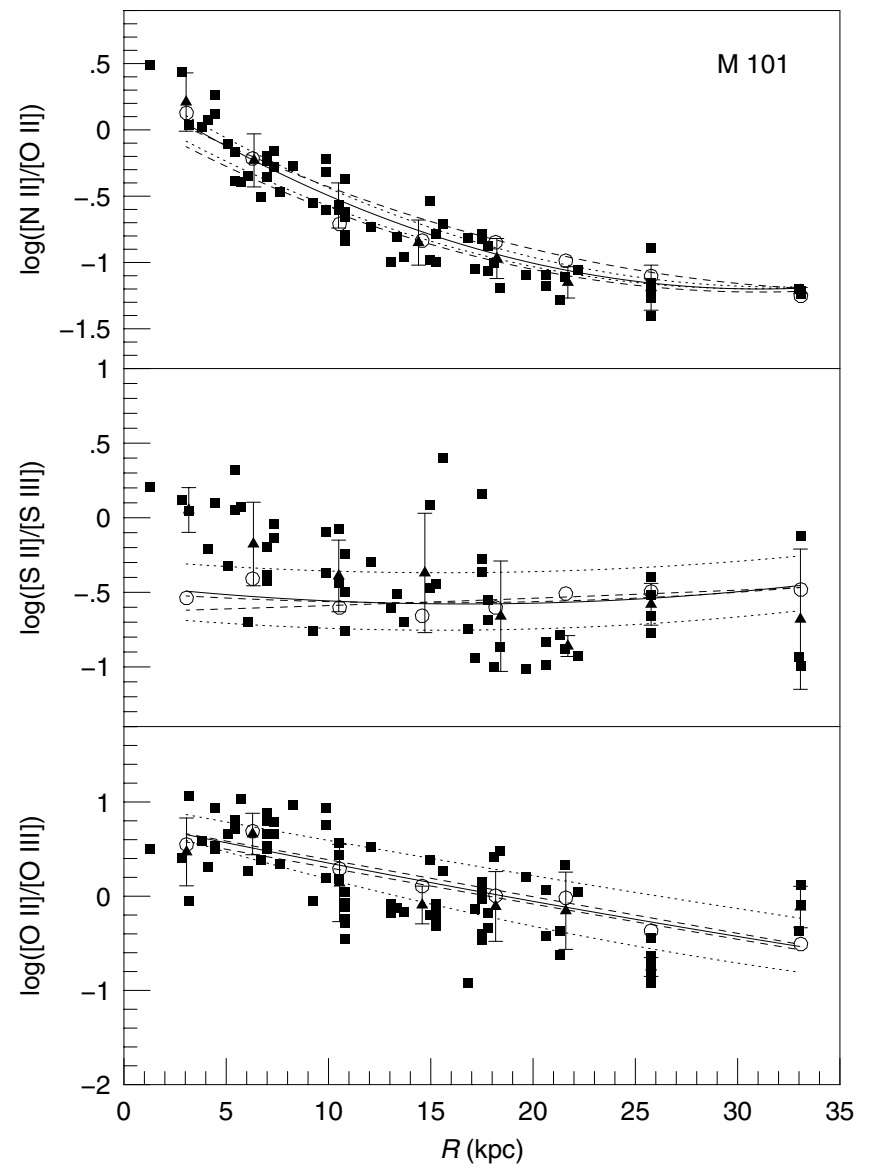

Fig. 1. [N II]/[O II], [S II]/[S III], and [O II]/[O III] ratios vs. galactocentric distance $R$ for M 101. Squares represent the observational data. Triangles represent the observed line ratio and galactocentric distance mean values within eight equally spaced segments of the galactic radius, and the error bars correspond to the standard deviations of the line ratios in each interval of radius. The open circles correspond to the line ratios of our best models, and the solid line represents a second degree polynomial fitting of them. The dashed and dotted lines correspond to the polynomial fitting of the line ratios predicted by models with the same parameters as our best models but with the abundances and the ionization parameter changed by 0.15 and $0.25 \mathrm{dex}$, respectively.

The N/O gradient via $P$-method derived by Pilyugin et al. (2004) is also shown in Fig. 3.

A linear regression in the our oxygen estimates yields $12+\log (\mathrm{O} / \mathrm{H})=(-0.035 \pm 0.002) R(\mathrm{kpc})+(9.15 \pm 0.04)$, consistent with the oxygen estimations obtained by Evans (1986). Our oxygen gradient yields a value of $12+\log (\mathrm{O} / \mathrm{H})=8.88$ for the H335 (Searle 5) nebula, similar to the one derived by Kinkel \& Rosa (1994). However, our O/H abundances are larger than those obtained via direct measurements (e.g. the ones by Kennicutt et al. 2003) by a factor of $0.1-0.3$ dex. As can be seen in Fig. 3, the gradient obtained via the $P$-method represents the variation of the direct abundances along the M 101 disk very well, and it is shallower than the other gradients. Similar agreement between the $P$-method and direct abundance estimates have also been found recently by Cedrés et al. (2004) and by Kennicutt et al. (2003) in M 101. The gradient derived via $R_{23}$ is quite similar to our gradient.

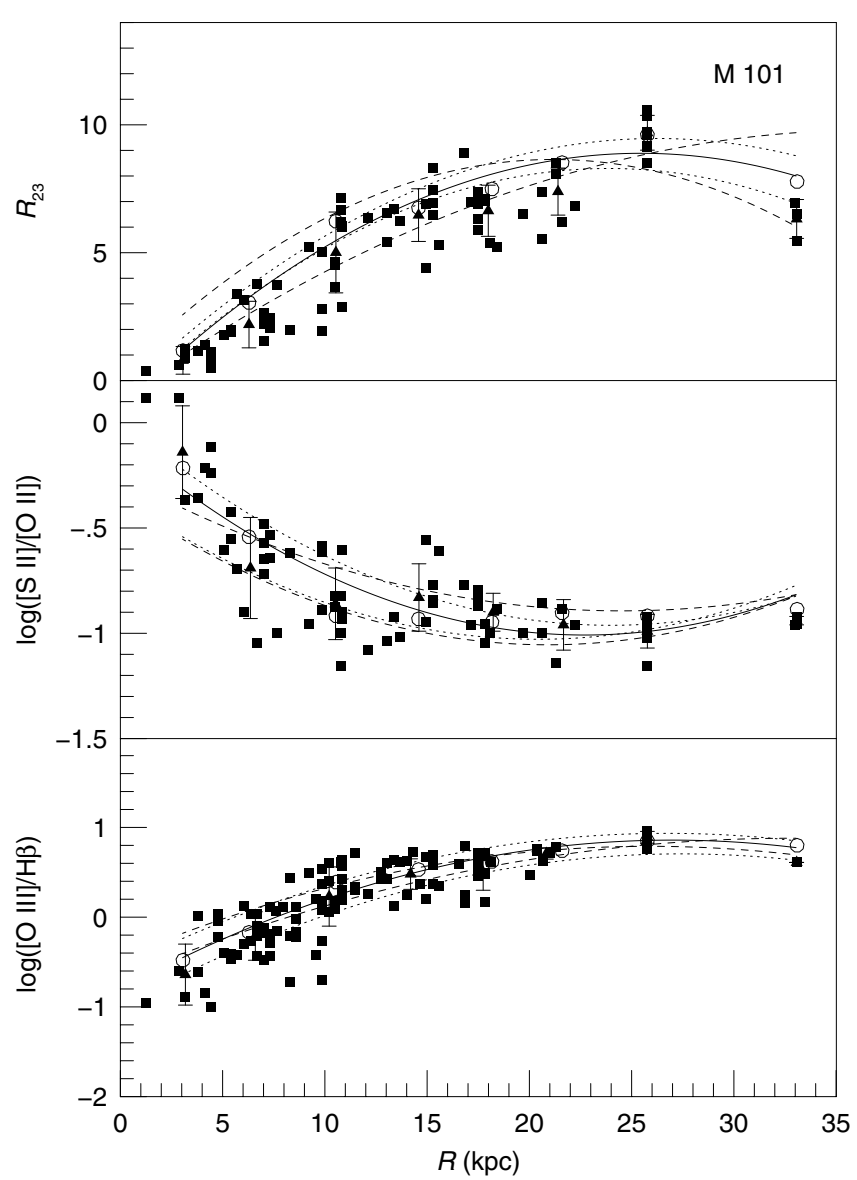

Fig. 2. Same as Fig. 1 but for $R_{23}$, [S II]/[O II] and [O III]/H $\beta$.

Our inferred results for the N/O ratio match the ones obtained from both direct measurements and the $P$-method, and they were fitted by $\log (\mathrm{N} / \mathrm{O})=(-0.024 \pm 0.004) R(\mathrm{kpc})-$ $(0.73 \pm 0.06)$. The N/O values found by Evans (1986) are about 0.1 dex lower than ours.

Our predictions marginally suggest an $\mathrm{S} / \mathrm{O}$ gradient represented by $\log (\mathrm{S} / \mathrm{O})=(-0.005 \pm 0.003) R(\mathrm{kpc})-(1.53 \pm 0.05)$. However, as can be noted in Fig. 1, our models failed to match the observed $[\mathrm{S} \mathrm{II}] /[\mathrm{S} \mathrm{III}]$ line ratios at high metallicities, which makes the $\mathrm{S} / \mathrm{O}$ ratio derived by us for this region very uncertain.

\subsection{NGC 1365}

In Figs. 4 and 5 the observational line ratio gradients and the results of our models for NGC 1365 are shown. An angular scale $1^{\prime \prime}=88$ pc (Roy \& Walsh 1997) was assumed. Unfortunately, due to the generally low level of excitation in NGC 1365, no direct measurements of electron temperature are available in the literature, so only empirical methods were used to derive abundances. Figure 6 shows our abundance results, as well as (i) $\mathrm{O} / \mathrm{H}$ and N/O ratios calculated by Pilyugin et al. (2004) via the $P$-method for $R<20 \mathrm{kpc}$, (ii) the abundance ratios obtained by Pagel et al. (1979) and Alloin et al. (1981) deriving electron temperatures from the $T_{\mathrm{e}}$ vs. $R_{23}$ empirical calibration by Pagel et al. (1979), and (iii) the $\mathrm{O} / \mathrm{H}$ gradient derived by Roy \& Walsh (1997) using the $R_{23}$ vs. O/H calibration from Edmunds \& Pagel (1984). 


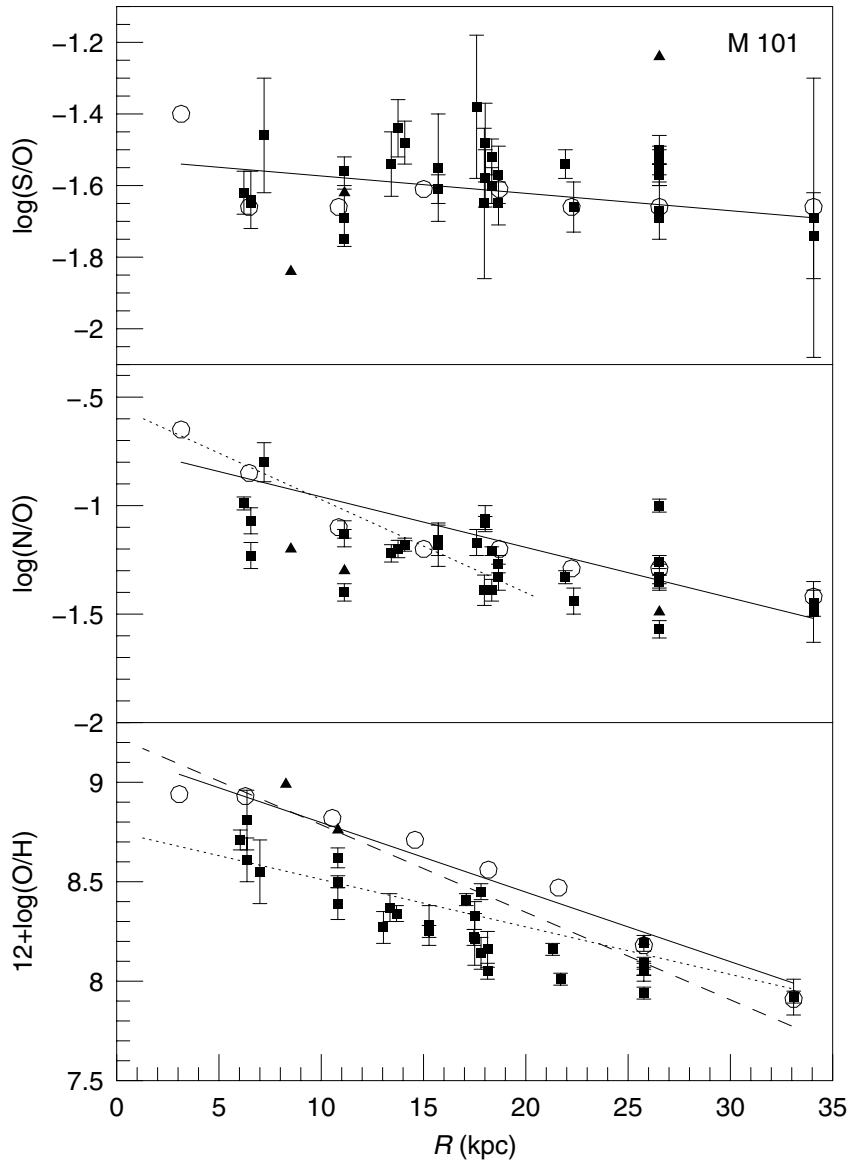

Fig. 3. Gradients of $12+\log (\mathrm{O} / \mathrm{H}), \mathrm{N} / \mathrm{O}$, and $\mathrm{S} / \mathrm{O}$ in $\mathrm{M} 101$. The solid lines are linear regressions of our best models represented by open circles. Middle panel: dotted line represents N/O gradient via $P$-method obtained by Pilyugin et al. (2004). Bottom panel: dashed and dotted lines represent $\mathrm{O} / \mathrm{H}$ gradient via $R_{23}$ and $P$-method obtained by Pilyugin (2001b). The filled squares represent the abundance ratios obtained via direct measurements of electron temperature collected from the literature and the triangles represent the abundance estimated by Evans (1986).

Roy \& Walsh (1997) found a break in the O/H radial distribution. For $R<16.9 \mathrm{kpc}$ they derived a gradient of $\Delta \log (\mathrm{O} / \mathrm{H}) / \Delta R=-0.05 \mathrm{dex} \mathrm{kpc}^{-1}$, while for $R>16.9 \mathrm{kpc}$ they found a constant oxygen abundance of $12+\log (\mathrm{O} / \mathrm{H})=8.65$. As can be seen in Fig. 6, the $\mathrm{O} / \mathrm{H}$ gradient derived by us does not show any evidence of an abundance break in NGC 1365. We found a smooth gradient represented by $12+\log (\mathrm{O} / \mathrm{H})=$ $(-0.010 \pm 0.001) R(\mathrm{kpc})+(8.97 \pm 0.01)$. Recently, Pilyugin (2003b) also contested the existence of an abundance break in NGC 1365. He reported that, although $\mathrm{O} / \mathrm{H}$ estimates derived by the $R_{23}$ index and by the $P$-method can show an oxygen abundance gradient break in the NGC 1365 disk, this result may be spurious since these methods yield incorrect $\mathrm{O} / \mathrm{H}$ values, once the abundances at the break region are close to the turnover point of the oxygen calibrations and also due to the low excitation of the nebulae. However, the existence of abundance breaks in barred spiral galaxies has been predicted in numerical simulations by Friedly et al. (1994) and by Friedly \& Benz (1995), and it has been interpreted as an indicator of the presence of young bars in this type of galaxy (Roy \& Walsh 1997; Friedli 1999; Considère et al. 2000). Another theoretical support was presented by Mishurov et al. (2002), who showed that bimodal abundance gradients can be a consequence of the existence of a corotation resonance in spiral arms. As pointed out by Pilyugin (2003b) the oxygen abundances in NGC 1365 will only be confirmed via direct measurements.

The $\mathrm{O} / \mathrm{H}$ abundances derived from our models are overestimated in relation to the ones obtained via the $P$-method by a factor of about 0.4 dex. The $\mathrm{O} / \mathrm{H}$ determinations derived by Alloin et al. (1981) and by Pagel et al. (1979) agree qualitatively with our results. The abundances for the three innermost $\mathrm{H}$ II regions present a very large scatter $(\approx 0.8 \mathrm{dex})$. These regions have unusual emission-line intensities when compared with outer regions. This behavior can be the signature of a secondary ionization mechanism, probably due to the hardionizing radiation coming from the NGC 1365 active nuclei. The use of a general oxygen calibration can yield unreliable abundance values for this type of object (Evans \& Dopita 1987), so we did not use the line ratios of these objects in the average computation of the data.

Figure 6 shows that the N/O results obtained from our models and from the $P$-method are consistent. We found an N/O gradient of $\log (\mathrm{N} / \mathrm{O})=(-0.015 \pm 0.003) R(\mathrm{kpc})-(0.68 \pm 0.05)$. On the other hand, the N/O values by Alloin et al. (1981) and by Pagel et al. (1979) are quite independent of galactocentric distance; and with a mean value of $\log (\mathrm{N} / \mathrm{O})=-1.10$, they are up to 0.5 dex lower than our results. These authors estimated the $\mathrm{N} / \mathrm{O}$ abundance using the $[\mathrm{N} \mathrm{II}] /[\mathrm{O}$ II $]$ ratio and a calibration of the electron temperature $T_{\mathrm{e}}$ with the $R_{23}$ and [N II]/[O III] line ratios, which can yield uncertain $T_{\mathrm{e}}$ values and consequently inaccurate $\mathrm{N} / \mathrm{O}$ ratios (Pilyugin 2001a). Moreover, a variation in the value of $[\mathrm{N} \mathrm{II}] /[\mathrm{O}$ II $]$ does not necessarily follow a change in the $\mathrm{N} / \mathrm{O}$ abundance. There is also a dependence of the $[\mathrm{N}$ II $] /[\mathrm{O}$ II $]$ ratio on $T_{\mathrm{e}}$ (Pagel et al. 1979), which should be taken into account in the derivation of N/O (Pilyugin et al. 2003).

Our models suggest a constant $\mathrm{S} / \mathrm{O}$ value of $\log (\mathrm{S} / \mathrm{O})=$ $-1.51 \pm 0.02$. The $T_{\text {eff }}$ parameter showed a systematic behavior following the relation $T_{\text {eff }}\left(10^{4} \mathrm{~K}\right)=(0.019 \pm 0.002) R(\mathrm{kpc})+$ $(3.77 \pm 0.03)$, and $\log U$ ranged from -2.3 to -2.8 .

\subsection{NGC 925}

Figures 7 and 8 show both the observational data for H II regions in the barred spiral galaxy NGC 925 and our result models plotted against the galactocentric distance $R$. An angular scale of $1^{\prime \prime}=34.3 \mathrm{pc}$ (Martin \& Roy 1994) was assumed.

Figure 9 shows the $\mathrm{O} / \mathrm{H}$ abundance gradient found by us, the $\mathrm{O} / \mathrm{H}$ estimates obtained by Martin \& Roy (1994) using the $\mathrm{O} / \mathrm{H}$ indicator $[\mathrm{O} \mathrm{III}] / \mathrm{H} \beta$, the ones by van Zee et al. (1998) obtained both from direct abundance determinations for two H II regions only and from a comparison with a grid of photoionization models by McGaugh (1991) for other objects, and finally the $\mathrm{O} / \mathrm{H}$ gradient derived by Pilyugin et al. (2004) via the $P$-method for $R<5.6 \mathrm{kpc}$. Our oxygen gradient, represented by $12+\log (\mathrm{O} / \mathrm{H})=(-0.016 \pm 0.003) R(\mathrm{kpc})+(8.76 \pm 0.02)$, yields 
$\mathrm{O} / \mathrm{H}$ values higher by 0.2 dex than the ones derived by Martin \& Roy (1994) and van Zee et al. (1998) and higher by 0.2 to 0.5 dex than the ones via $P$-method. As can be seen in Fig. 8 , the flattening in the $R_{23}$ oxygen indicator at $R \approx 6.0 \mathrm{kpc}$ could be indicating the presence of an oxygen break in NGC 925 . However, the $\mathrm{H}$ II regions beyond this limit have abundances lower than $12+\log (\mathrm{O} / \mathrm{H})=8.2$ (Pilyugin et al. 2004), which can produce a false bend in the slope of the oxygen gradient obtained from the $\mathrm{O} / \mathrm{H}$ calibrations (Pilyugin 2003b).

Also in Fig. 9, the N/O and S/O gradients obtained from our models are compared with those derived by van Zee et al. (1998) and by Pilyugin et al. (2004) using the $P$-method. Our models yielded N/O values consistent with the ones derived by Pilyugin et al. (2004), but they are somewhat larger than the ones from van Zee et al. (1998). The $\mathrm{N} / \mathrm{O}$ gradient derived from our models is given by $\log (\mathrm{N} / \mathrm{O})=(-0.029 \pm 0.003) R(\mathrm{kpc})-$ $(1.10 \pm 0.04)$. As can be seen in Fig. 7, the $[\mathrm{N} \mathrm{II}] /[\mathrm{O}$ II] line ratios are about constant at $R>5 \mathrm{kpc}$, from which an artificial abundance break could be inferred if we used general abundance calibrations as, for example, the $[\mathrm{N}$ II $] /[\mathrm{O}$ II $]$ vs. O/H calibration by Kewley \& Dopita (2002).

We found a flattened $\mathrm{S} / \mathrm{O}$ gradient of $\log (\mathrm{S} / \mathrm{O})=(-0.007 \pm$ $0.003) R(\mathrm{kpc})-(1.47 \pm 0.02)$, which yields $\mathrm{S} / \mathrm{O}$ values consistent with the ones obtained by van Zee et al. (1998); however, for the outermost $\mathrm{H}$ II region, the $\mathrm{S} / \mathrm{O}$ value obtained by van Zee et al. (1998) is lower than the one derived by us by about 0.4 dex. For this object the [S III] $\lambda 6312$ line was not detected, so van Zee et al. (1998) used an average value for the ionization correction factor to derive the sulfur abundance, which can make this value doubtful. A $T_{\text {eff }}$ gradient represented by $T_{\text {eff }}\left(10^{4} \mathrm{~K}\right)=(0.027 \pm 0.006) R(\mathrm{kpc})+(3.97 \pm 0.03)$ and $\log U$ ranging from -2.4 to -3.0 were derived from our models.

Castellanos et al. (2002) observed four inner H II regions in NGC 925, in the distance range of $0.17-1.4 \mathrm{kpc}$, namely CDT1, CDT2, CDT3, and CDT4. Using an $\mathrm{S}_{23}$ vs. $\mathrm{O} / \mathrm{H}$ calibration, $\mathrm{S}_{23}=([\mathrm{S}$ II $] \lambda \lambda 6716,6731+$ $[\mathrm{S}$ III $] \lambda \lambda 9069,9532) / \mathrm{H} \beta$, they derived $12+\log (\mathrm{O} / \mathrm{H})=8.52$, $8.71,8.50$, and 8.41 , respectively, for these regions. These values are lower than the ones we derived by about 0.3 dex. However, Castellanos et al. (2002) noted that their O/H determinations can be somewhat uncertain, because the measured values of $S_{23}$ fall near the reversal point of the abundance calibration relationship. Using detailed photoionization models based on the NLTE atmosphere models of Mihalas (1972), Castellanos et al. (2002) obtained for CDT1 and CDT4 mean values of $12+\log (\mathrm{O} / \mathrm{H})=8.7, \log (\mathrm{N} / \mathrm{O})=-0.97$, $\log (\mathrm{S} / \mathrm{O})=-1.71$, and $T_{\text {eff }}=36500 \mathrm{~K}$. The $T_{\text {eff }}$ and N/O values are about the same as in our results, while the $\mathrm{S} / \mathrm{O}$ ratio is lower than our predictions by 0.25 dex.

\subsection{NGC 1073}

Figure 10 shows the observational gradients of $[\mathrm{N} \mathrm{II]} /[\mathrm{O}$ III] and $[\mathrm{O} \mathrm{III}] / \mathrm{H} \beta$ and our model results for NGC 1073 . The angular scale $1^{\prime \prime}=55.4$ pc (Martin \& Roy 1994) was assumed. The four innermost points represent H II regions located in the bar of NGC 1073, which may have a secondary ionization source (Martin \& Roy 1994), so our analysis did not extend to these points. The observational data do not include the emission lines needed for sulfur abundance determinations, so all models were built considering a solar $\mathrm{S} / \mathrm{O}$ ratio. We derive an effective temperature gradient represented by $T_{\text {eff }}\left(10^{4} \mathrm{~K}\right)=$ $(0.073 \pm 0.004) R(\mathrm{kpc})+(3.89 \pm 0.02)$ and a mean ionization parameter of $\log U \approx-2.80 \pm 0.05$. The absence of an observational $[\mathrm{O} \mathrm{II}] /[\mathrm{O} \mathrm{III}]$ line ratio can make these results somewhat uncertain, because this ratio is strongly dependent on those two parameters (Dors \& Copetti 2003; Morisset et al. 2004).

In Fig. 11 our $\mathrm{O} / \mathrm{H}$ gradient is compared with the one obtained by Martin \& Roy (1994) via a [O III]/H $\beta$ vs. O/H calibration. For NGC 1073 we derive an $\mathrm{O} / \mathrm{H}$ gradient of $12+$ $\log (\mathrm{O} / \mathrm{H})=(-0.048 \pm 0.012) R(\mathrm{kpc})+(8.74 \pm 0.06)$, very consistent with the one derived by Martin \& Roy (1994).

We obtained a mean value of $\log (\mathrm{N} / \mathrm{O})=-1.05 \pm 0.05$ and no systematic variation of N/O with $R$ was found. The N/O abundances obtained for H II regions in NGC 1073 are larger than the ones for similar oxygen abundance objects found in other galaxies. In order to prove that this overabundance of nitrogen is not an artefact of our models, we use Fig. 12 to plot $[\mathrm{N} \mathrm{II]} /[\mathrm{O} \mathrm{III}]$ vs. [O III $] / \mathrm{H} \beta$, in order to show that the [N II] lines measured by Martin \& Roy (1994) in H II regions of NGC 1073 are relatively stronger than the ones obtained in 34 other spiral galaxies by Bresolin et al. (1999), van Zee et al. (1998), Edmunds \& Pagel (1984), Deharveng et al. (1988), Henry et al. (1992), and Ferguson et al. (1998). England et al. (1990) suggested that NGC 1073 may have suffered an interaction in the past, and Martin \& Roy (1994) pointed out that the intense star formation activity in its bar can be a signature of this interaction. As a result, the overabundance of nitrogen in the NGC 1073 disk could have possibly been yielded by a distinct chemical evolution due to an encounter with another galaxy. Further abundance studies based on emission-line measurements obtained with a more reliable observational technique than the one used by Martin \& Roy (1994) are necessary to confirm the N/O overabundance in H II regions of NGC 1073.

\section{Discussion}

Our work supports the idea that the observed gradients of emission-line intensities of $\mathrm{H}$ II regions in the disks of spiral galaxies are mainly caused by a negative gradient of metal abundance and, secondarily, by an increase in the effective temperature of ionizing stars with the galactocentric distance. Our models indicate that the scatter in the observed line ratios at a given galactocentric distance can be explained by a variation of \pm 0.15 dex in abundance or \pm 0.25 dex in the ionization parameter. This metallicity variation is the accuracy with which the oxygen abundance of $\mathrm{H}$ II regions may be derived from photoionization model sequences (Evans 1986).

For the four galaxies studied we found a positive $T_{\text {eff }}$ gradient of $\Delta T_{\text {eff }} / \triangle R=(400 \pm 112) \mathrm{K} \mathrm{kpc}^{-1}$ that indicates an anticorrelation between $T_{\text {eff }}$ and metallicity. Many previous studies have reached the same conclusion (e.g., Shields \& Tinsley 1976; Stasińska 1980; Vilchez \& Pagel 1988; Campbell 1988; Bresolin et al. 1999; Dors \& Copetti 2003). However, recently 
the opposite trend has been supported by Morisset (2004), who when comparing observational diagnostic diagrams for mid-IR emission lines with photoionization model grids, found an increase in $T_{\text {eff }}$ with the metallicity in the Milky Way. Morisset (2004) argued that this is the correct conclusion if photoionization models use consistent metallicities for the stellar atmosphere and for the nebula.

We have not detected any systematic dependency of the ionization parameter on the galactocentric distance or metallicity. We did find ionization parameters in the range $-3.0 \leq$ $\log U \leq-2.3$. Analyzing abundance estimates across the disks of galaxies, Zaritsky et al. (1994) reached the same conclusion; however, Bresolin et al. (1999) found that for the most metal poor objects of their sample, the ionization parameter is about 4 times larger than in $\mathrm{H}$ II regions with solar metallicity.

Our $\mathrm{O} / \mathrm{H}$ estimates are larger than the ones obtained via direct temperature determinations and via $P$-method by a factor of 0.1-0.4 dex, with the difference increasing with $\mathrm{O} / \mathrm{H}$. Two opposite explanations can be proposed for this discrepancy. On one hand, abundances derived from the comparison of strong emission lines with photoionization models; (i) could possibly be overestimated when compared to direct determinations if these models are not realistic enough, (ii) do not treat all the relevant physical processes correctly, and (iii) use inaccurate atomic data, spheric geometry, etc. (Viegas 2002; Kennicutt et al. 2003). Recently using photoionization models, Garnett et al. (2004b) derived, for the H II region CCM 10 in M 51, metal abundances that are lower by a factor of 2-3 than those derived previously by Diaz et al. (1991), who used an older version of the Cloudy photoionization code than the one used by the earlier authors. So, progresses (on several fronts) in the development of photoionization codes could conciliate theoretical and direct metal determinations in the future. On the other hand, the direct abundance estimates could be underestimated by not taking the effects of electron temperature fluctuations into account. Ruiz et al. (2003) showed that, in order to match abundance from recombination lines with those from forbidden lines, it is necessary to consider a temperature fluctuation with relative variance of $t^{2}=0.056$, the parameter $t^{2}$ defined as in Peimbert (1967), which makes the abundances higher by $0.2-0.3$ dex than the ones with $t^{2}=0$. If we applied such correction over the direct oxygen abundances utilized in this paper, it would reconcile our predictions. However, theoretical and observational studies (e.g., Kingdon \& Ferland 1995; Krabbe \& Copetti 2002; Rubin et al. 2003) have up to now failed to predict or directly detect such high levels of electron temperature fluctuations. Strong evidence against the presence of temperature fluctuations in planetary nebulae and in H II regions have been also found by Liu et al. (2000, 2001, 2004) and Tsamis et al. (2003), respectively. In particular, Liu et al. (2000) derived abundances for the planetary nebula NGC 6153 from infrared emission lines (weakly sensitive to $T_{\mathrm{e}}$ ), which are consistent with the ones from optical and ultraviolet emission lines, and this does the temperature fluctuation hypothesis increasingly doubtful. Moreover, at least for solar metallicities, the reliability of direct temperature determinations in H II regions seems to be confirmed, since a good agreement has been found between oxygen abundance determinations for nebulae in the solar neighborhood and ones derived from observations of the weak interstellar OI $\lambda 1356$ line towards the stars (Pilyugin 2003a; Moss et al. 2002; Deharveng et al. 2000; Rolleston et al. 2000; Meyer et al. 1998).

Our results for $\mathrm{O} / \mathrm{H}$ abundances generally agree with the ones from empirical oxygen calibrations with $R_{23}$ and [O III]/H $\beta$ line ratio by Edmunds \& Pagel (1984), what is expected since these authors also use photoionization models in their calibrations. However, the flatter $\mathrm{O} / \mathrm{H}$ gradient obtained from our models for M 101 and NGC 1365 has important implications for empirical calibration of high abundances. Many studies have investigated the influence of physical parameters (e.g., $T_{\text {eff }}, U$, dust) on oxygen empirical calibrations (see McGaugh 1991; Henry 1993; Oey \& Kennicutt 1993; Kewley \& Dopita 2002), and the calibration for high metallicities has presented considerable variation along the years; so it is instructive to compare our results with calibrations suggested by other authors. The $[\mathrm{O} \mathrm{III}] / \mathrm{H} \beta$ ratio has been shown to give very crude oxygen abundances with uncertainties on the order of 0.5 dex (Kobulnicky et al. 1999), so it will not be used for any comparison. Figure 13 shows the $R_{23}$ vs. O/H relationship from our models, the calibrations by Edmunds \& Pagel (1984), Kobulnicky et al. (1999) and Pilyugin (2001a), and from directly estimated oxygen abundances (Bresolin et al. 2004; Kennicutt et al. 2003; Castellanos et al. 2002; Rayo et al. 1982; Torres-Peimbert et al. 1989; van Zee et al. 1998). Since most of the HII regions considered in this paper have metallicities that place them on the upper branch of this diagram, our discussion will be only for this part of the calibrations. The Kobulnicky et al. (1999) and Pilyugin (2001a) calibrations are parameterized in terms of a second parameter $y=\log ([\mathrm{O}$ III $](\lambda 4949+\lambda 5007) /[\mathrm{O}$ II $](\lambda 3726+\lambda 3729))$ and $P=([\mathrm{O}$ III $] \lambda 4949+\lambda 5007) / \mathrm{H} \beta / R_{23}$, respectively. We drew curves for these calibrations for the values of $y=-0.45,-1.0$ and $P=0.25,0.8$. The $R_{23}$ vs. $\mathrm{O} / \mathrm{H}$ calibration derived from our models is approximated well by

$12+\log (\mathrm{O} / \mathrm{H})=8.96-0.03 x-0.10 x^{2}-0.21 x^{3}-0.26 x^{4}$,

where $x=\log R_{23}$. Our predictions generally agree well with calibrations by Kobulnicky et al. (1999) and by Pilyugin (2001a) with $P=0.8$, appropriate for high excitation objects. However, our calibration overestimates the oxygen abundance by a factor of $0.1-0.4$ dex compared to direct estimates and to the Pilyugin (2001a) method with $P=0.25$, appropriate for low excitation objects.

As for sulfur, there is no clear consensus on the behavior of the S/O ratio across the galactic disks. Smith (1975), Shields \& Searle (1978), Torres-Peimbert et al. (1989), Garnett (1989), van Zee et al. (1998), Kennicutt et al. (2003), Izotov et al. (2004), and Bresolin et al. (2004) all found no evidence whatsoever for a dependence of S/O on either radius or oxygen abundance, while the existence of an anticorrelation between $\mathrm{S} / \mathrm{O}$ and $\mathrm{O} / \mathrm{H}$ has been supported by some other authors (e.g., Shaver et al. 1983; Vilchez et al. 1988; Diaz et al. 1991; Evans 1986). Considering uncertainties in the S/O gradients found, this work supports the idea that $\mathrm{S} / \mathrm{O}$ is about constant with $\mathrm{O} / \mathrm{H}$. The strong increase in $\mathrm{S} / \mathrm{O}$ with $\mathrm{O} / \mathrm{H}$ found for the innermost regions of the M 101 disk seems to be an artefact of the models 


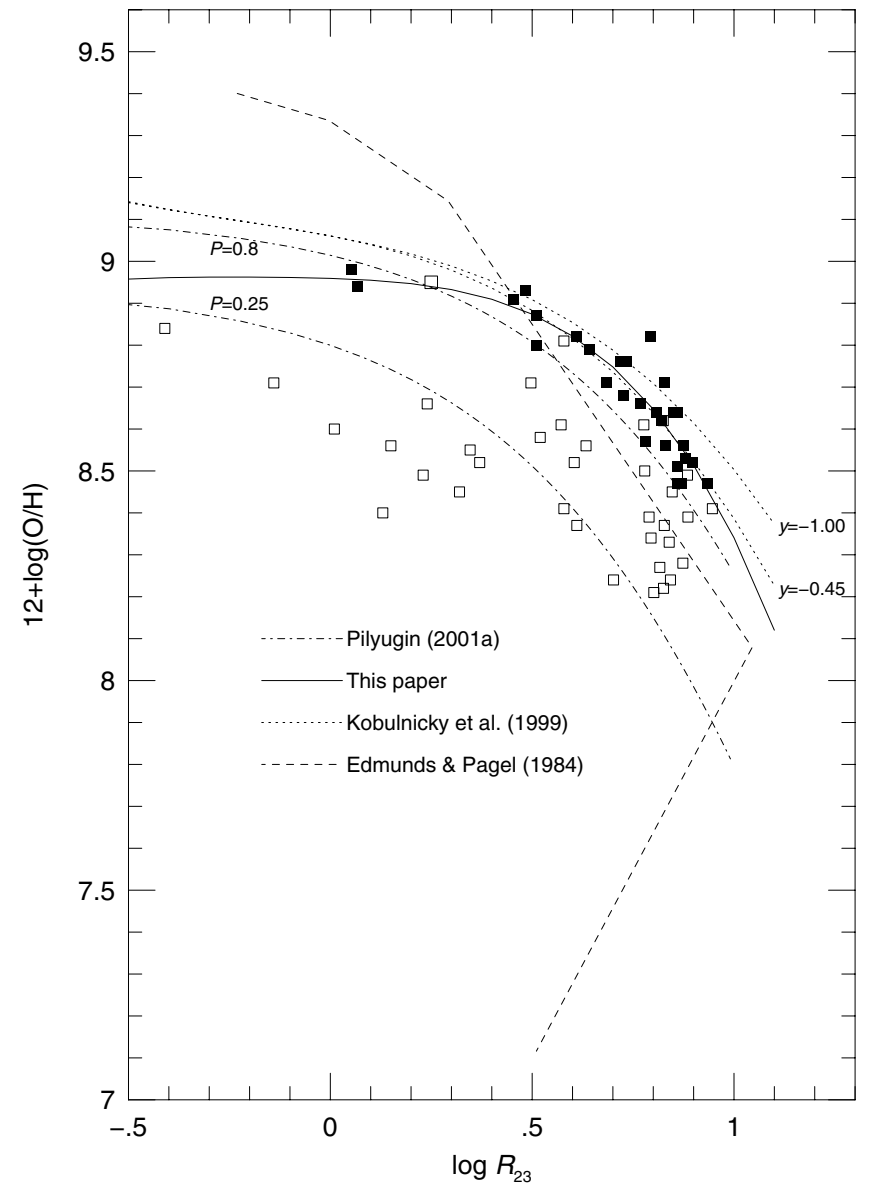

Fig. 13. Comparison of different oxygen abundance calibrations with $R_{23}$. The filled squares represent our models, and the open squares correspond to directly estimated oxygen abundances.

once they underestimate the $[\mathrm{S} \mathrm{II}] /[\mathrm{S} \mathrm{III}]$ ratios at high metallicities. Garnett (1989) also found that his models tend to underestimate that line ratio and suggested that this was due to uncertainties in stellar atmosphere models or in atomic data for sulfur. Geometrical differences between the models and nebulae or temperature fluctuations in nebulae may also be responsible for the discrepancy between the observed and predicted line ratios (Evans 1986). Clearly, a larger number of measurements of [S III] emission-line and direct S/O determinations, specially of nebulae with high abundances, will help to uncover a possible trend in the $\mathrm{S} / \mathrm{O}$ abundance ratio.

Unlike the $\mathrm{O} / \mathrm{H}$ abundances, $\mathrm{N} / \mathrm{O}$ ratios derived both from the direct and $P$-methods agree very well with the predictions of our models, which indicates that our procedure overestimates the $\mathrm{O} / \mathrm{H}$ and $\mathrm{N} / \mathrm{H}$ abundances by the same amount in comparison to the other two methods. In Fig. 14, an $\mathrm{O} / \mathrm{H}$ vs. N/O diagram, we confront our results with two different chemical evolution models proposed by Vila-Costas \& Edmunds (1993), one considering that the synthesis of nitrogen has both a primary and a secondary component relative to oxygen, and the other assuming that nitrogen is a purely secondary synthesis product. In general, our N/O estimates populate the permitted area defined by Vila-Costas \& Edmunds (1993). For the $12+\log (\mathrm{O} / \mathrm{H}) \leq 8.2$ found in our sample only at the outer parts

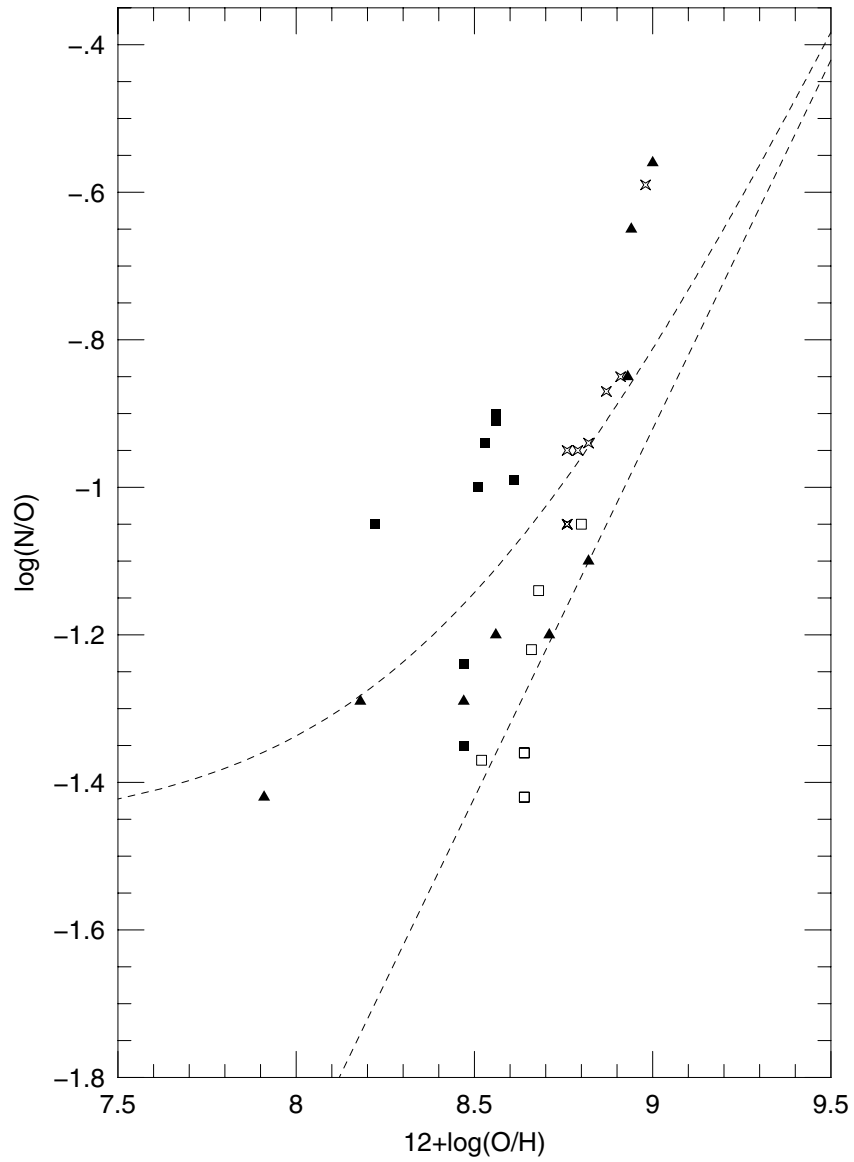

Fig. 14. $\log (\mathrm{N} / \mathrm{O})$ vs. $12+\log (\mathrm{O} / \mathrm{H})$. Dashed lines represent the chemical evolution models from Vila-Costas \& Edmunds (1993). The marks represent our results for M101 (₫), NGC 1073 (•), NGC 925 (口), and NGC $1365(\star)$.

of the M 101 disk, the contribution of the primary nitrogen synthesis becomes essential. For the regime of high metallicities $\left(Z>Z_{\odot}\right)$, the results for M 101 and NGC 1365 suggest that N/O has a steeper dependence on the oxygen abundance than that predicted by the models by Vila-Costas \& Edmunds (1993). A similar strong dependence of $\mathrm{N} / \mathrm{O}$ on $\mathrm{O} / \mathrm{H}$ at high abundances was found by Henry et al. (2000). NGC 1073 seems to be overabundant in nitrogen compared to the oxygen abundance, and these simple chemical evolution models do not agree with our results for this galaxy. More sophisticated models, including dynamical processes such as gas inflow and outflow, may be needed in the case of NGC 1073.

\section{Conclusions}

We built photoionization models in order to reproduce the gradients of emission-line intensity ratios for $\mathrm{H}$ II regions located along the disk of the normal spiral galaxy M 101 and in three barred spiral galaxies NGC 1365, NGC 925, and NGC 1073. Our results indicate a positive gradient of about $\Delta T_{\text {eff }} / \Delta R=(400 \pm 112) \mathrm{K} \mathrm{kpc}^{-1}$ for the stellar effective temperature in the galaxies considered. We found ionization parameters in the range $-3.0 \leq \log U \leq-2.3$. No clear systematic variation was found in the ionization parameter with the 
galactocentric distance. Our oxygen abundances are overestimated when compared to direct estimates based on measurements of the electron temperature by $0.1-0.4$ dex. This discrepancy has been either attributed to uncertainties in photoionization models or considered as evidence of large temperature fluctuations in $\mathrm{H}$ II regions.

No S/O gradient was found in the galaxies studied; however, our prediction for $\mathrm{S} / \mathrm{O}$ ratios in the regime of high abundances may be uncertain, since our models failed to reproduce the $[\mathrm{S} \mathrm{II}] /[\mathrm{S} \mathrm{III}]$ ratio. Our results for NGC 1365 are consistent with the ones obtained by Pilyugin (2003b), who has contested the existence of an abundance break in this galaxy found by Roy \& Walsh (1997). For NGC 925, our model results show that the flattening in the $R_{23}$ gradient is not necessarily due to a break in the $\mathrm{O} / \mathrm{H}$ abundance gradient. The $\mathrm{N} / \mathrm{O}$ vs. $\mathrm{O} / \mathrm{H}$ relation derived from our models agrees in part with the chemical evolution models proposed by Vila-Costas \& Edmunds (1993), which consider that the synthesis of nitrogen have both primary and secondary components. However, we found a steeper dependence of $\mathrm{N} / \mathrm{O}$ with $\mathrm{O} / \mathrm{H}$ at high metallicities than the early chemical models predict. Our results also indicate an overabundance of nitrogen in NGC 1073.

Acknowledgements. We thank Dr. Gary Ferland for making the Cloudy programme available as well as the anonymous referee for many useful comments. We gratefully acknowledge Dr. Claus Leitherer for help with STARBURST99 and to the LSC (Laboratório de Sistemas de Computação, UFSM), where the calculations were performed. Many thanks also to Dr. Donald Garnett by his valuable suggestions. This work was supported by the Brazilian institutions CAPES and FAPERGS.

\section{References}

Allende Prieto, C., Lambert, D. L., \& Asplund, M. 2001, ApJ, 556, 63 Alloin, D., Collin-Souffrin, S., Joly, M., \& Vigroux, L. 1979, A\&A, 78, 200

Alloin, D., Edmunds, M. G., Lindblad, P. O., \& Pagel, B. E. J. 1981, A\&A, 101, 377

Bresolin, F., Kennicutt, R. C., \& Garnett, D. R. 1999, ApJ, 510, 104

Bresolin, F., Garnett, D. R., \& Kennicutt, R. C. 2004, ApJ, 615, 228

Campbell, A. 1988, ApJ, 335, 644

Castellanos, M., Diaz, A. I., \& Terlevich, E. 2002, MNRAS, 329, 315

Cedrés, B., Urbaneja, M. A., \& Cepa, J. 2004, A\&A, 422, 511

Considère, S., Coziol, R., Contini, T., \& Davoust, E. 2000, A\&A, 356, 89

Deharveng, L., Caplan, J., Leuquex, J., et al. 1988, A\&AS, 73, 407

Deharveng, L., Peña, M., Caplan, J., \& Costero, R. 2000, MNRAS, 311,329

Díaz, A. I., Terlevich, E., Vilchez, J. M., Pagel, B. E. J., \& Edmunds, M. G. 1991, MNRAS, 253, 245

Dors, O. L., \& Copetti, M. V. F. 2003, A\&A, 404, 969

Edmunds, M. G., \& Pagel, B. E. J. 1984, MNRAS, 211, 507

Edmunds, M. G., \& Roy, J. R. 1993, MNRAS, 261, L17

England, M. N., Gottesman, S. T., \& Hunter, J. H. 1990, ApJ, 348, 456

Evans, I. N. 1986, ApJ, 309, 544

Evans, I. N., \& Dopita, M. A. 1987, 319, 622

Ferland, G. J. 2002, Hazy, a brief introduction to Cloudy 96.03, Univ. Kentucky, Dept. Phys., Astron. internal report
Ferguson, A. M. N., Gallagher, J. S., \& Wyse, R. F. G. 1998, AJ, 116, 673

Friedli, D. 1999, in ASP Conf. Ser., ed. J. E. Beckman, \& T. J. Mahoney, 187, 88

Friedli, D., \& Benz, W. 1995, 301, 649

Friedli, D., Benz, W., \& Kennicutt, R. C. 1994, ApJ, 430, 105

Garnett, D. R. 1989, ApJ, 345, 282

Garnett, D. R., \& Kennicutt, R. C. 1994, ApJ, 426, 123

Garnett, D. R., Dufour, R. J., Peimbert, M., et al. 1995, ApJ, 449, 77

Garnett, D. R., Shields, G. A., Peimbert, M., et al. 1999, ApJ, 513, 168

Garnett, D. R., Kennicutt, R. C., \& Bresolin, F. 2004a, ApJ, 607, L21

Garnett, D. R, Edmunds, M. G., Henry, R. B. C., Pagel, B. E. J., \& Skillman, E. D. 2004b, AJ, 128, 2772

Grevesse, N., \& Sauval, A. 1998, Space Sci. Rev., 85, 161

Henry, R. B. C. 1993, MNRAS, 261, 306

Henry, R. B. C., Pagel, B. E. J., Lasseter, D. F., \& Chincarini, G. L. 1992, MNRAS, 258, 321

Henry, R. B. C., \& Howard, J. W. 1995, ApJ, 438, 170

Henry, R. B. C., Edmunds, M. G., \& Koppen, J. 2000, ApJ, 541, 660

Holweger, H. 2001, in Solar and Galactic Composition, ed. R. F. Wimmer-Scweingruber (New York Springer), AIP Conf. Ser., 598, 23

Hubeny, I., \& Lanz, T. 1995, ApJ, 439, 875

Izotov, Y. I., Stasińska, G., Guseva, N. G., \& Thuan, T. X. 2004, A\&A, 415,87

Kewley, L. J., \& Dopita, M. A. 2002, ApJS, 142, 35

Kennicutt, R. C., \& Garnett, D. R. 1996, ApJ, 456, 504

Kennicutt, R. C., Bresolin, F., French, H., \& Martin, P. 2000, ApJ, 537, 589

Kennicutt, R. C., Bresolin, F., \& Garnett, D. R. 2003, ApJ, 591, 801

Kingdon, J. B., \& Ferland, G. J. 1995, ApJ, 450, 691

Kinkel, U., \& Rosa, M. R. 1994, A\&A, 282, L37

Kobulnicky, H. A., Kennicutt, R. C., \& Pizagno, J. L. 1999, ApJ, 514, 544

Krabbe, A. C., \& Copetti, M. V. F. 2002, A\&A, 387, 295

Lanz, T., \& Hubeny, I. 2003, ApJS, 146, 417

Liu, X.-W., Storey, P. J., Barlow, M. J., et al. 2000, MNRAS, 312, 585

Liu, X.-W., Luo, S.-G., Barlow, M. J., Danziger, I. J., \& Storey, P. J. 2001, MNRAS, 327, 141

Liu, Y., Liu, X.-W., Barlow, M. J., \& Luo, S.-G. 2004, 353, 1251

Leitherer, C., Schaerer, D., Goldader, J. D., et al. 1999, ApJS, 123, 3

McGaugh, S. S. 1991, ApJ, 380, 140

Martin, P., \& Roy, J. R. 1994, ApJ, 424, 599

Martin, P., \& Roy, J. R. 1995, ApJ, 445, 161

Meyer, D. M., Jura, M., \& Cardelli, J. A. 1998, ApJ, 493, 222

Mihalas, D. 1972, Non-LTE Model Atmospheres for B and O stars, NCAR TN/STR-76

Mishurov, Yu. N., Lépine, J. R. D., \& Achorova, I. A. 2002, ApJ, 571, L113

Morisset, C. 2004, ApJ, 601, 858

Morisset, C., Schaerer, D., Martín-Hernández, N. L., et al. 2002, A\&A, 386, 558

Morisset, C., Schaerer, D., Bouret, J. C., \& Martins, F. 2004, A\&A, 415, 577

Moos, H. W., Sembach, K. R., Vidal-Madjar, A., et al. 2002, ApJS, 140,3

Oey, M. S., \& Kennicutt, R. C. 1993, ApJ, 411, 137

Pagel, B. E. J., Edmunds, M. G., Blackwell, D. E., Chun, M. S., \& Smith, G. 1979, MNRAS, 189, 95

Pagel, B. E. J., Simonson, E. A., Terlevich, R. J., \& Edmunds, M. G. 1992, MNRAS, 255, 325

Peimbert, M. 1967, ApJ, 150, 825

Pilyugin, L. S. 2001a, A\&A, 369, 594 
Pilyugin, L. S. 2001b, A\&A, 373, 56

Pilyugin, L. S. 2003a, A\&A, 399, 1003

Pilyugin, L. S. 2003b, A\&A, 397, 109

Pilyugin, L. S., Thuan, T. X., \& Vilchez, J. M. 2003, A\&A, 397, 487

Pilyugin, L. S., Contini, T., \& Vilchez, J. M. 2004, A\&A, 423, 427

Rayo, J. F., Peimbert, M., \& Torres-Peimbert, S. 1982, ApJ, 255, 1

Rolleston, W. R. J., Smartt, S. J., Dufton, P. L., \& Ryans, R. S. I. 2000, A\&A, 363, 537

Roy, J. R., \& Walsh, J. R. 1997, MNRAS, 288, 715

Rubin, R. H., Martin, P. G., Dufour, R. J., et al. 2003, MNRAS, 340, 362

Ruiz, M. T., Peimbert, A., Peimbert, M., \& Esteban, C. 2003, ApJ, 595,247

Searle, L. 1971, ApJ, 168, 327

Scowen, P. A., Dufour, R. J., \& Hester, J. J. 1992, AJ, 104, 92

Shields, G. A. 1986, PASP, 98, 1072

Shields, G. A., \& Tinsley, B. M. 1976, ApJ, 203, 66

Shields, G. A., \& Searle, L. 1978, ApJ, 222, 821

Smith, H. E. 1975, ApJ, 199, 591

Stasińska, G. 1980, A\&A, 84, 320
Shaver, P. A., McGee, R. X., Newton, L. M., Danks, A. C., \& Pottasch, S. R. 1983, MNRAS, 204, 53

Tsamis, Y. G., Barlow, M. J., Liu, X.-W., Danziger, I. J., \& Storey, P. J. 2003, MNRAS, 338, 687

Torres-Peimbert, S., Peimbert, M., \& Fierro, J. 1989, ApJ, 345, 186 van Hoof, P. A. M., Weingartner, J. C., Martin, P. G., Volk, K., \& Ferland, G. J. 2001, in Challenges of Photoionized Plasmas, ed. G. Ferland \& D. Savin (San Francisco: ASP), ASP Conf Ser., 247, 363

van Zee, L., Salver, J. J., Haynes, M. P., O’Donoghe, A. A., \& Balonek, T. J. 1998, AJ, 116, 2805

Viegas, S. M. 2002, RMxAc, 12, 219

Vilchez, J. M., \& Pagel, B. E. J. 1988, MNRAS, 231, 257

Vilchez, J. M., Pagel, B. E. J., Diaz, A. I., Terlevich, E., \& Edmunds, M. G. 1988, MNRAS, 235, 633

Vila-Costas, M. B., \& Edmunds, M. G. 1992, MNRAS, 259, 121

Vila-Costas, M. B., \& Edmunds, M. G. 1993, MNRAS, 265, 199

Zaritsky, D. 1992, ApJ, 390, L73

Zaritsky, D., Kennicutt, R. C., \& Huchra, J. P. 1994, ApJ, 420, 87 
O. L. Dors Jr. and M. V. F. Copetti: Abundance gradients in barred galaxies, Online Material p 1

\section{Online Material}


O. L. Dors Jr. and M. V. F. Copetti: Abundance gradients in barred galaxies, Online Material p 2

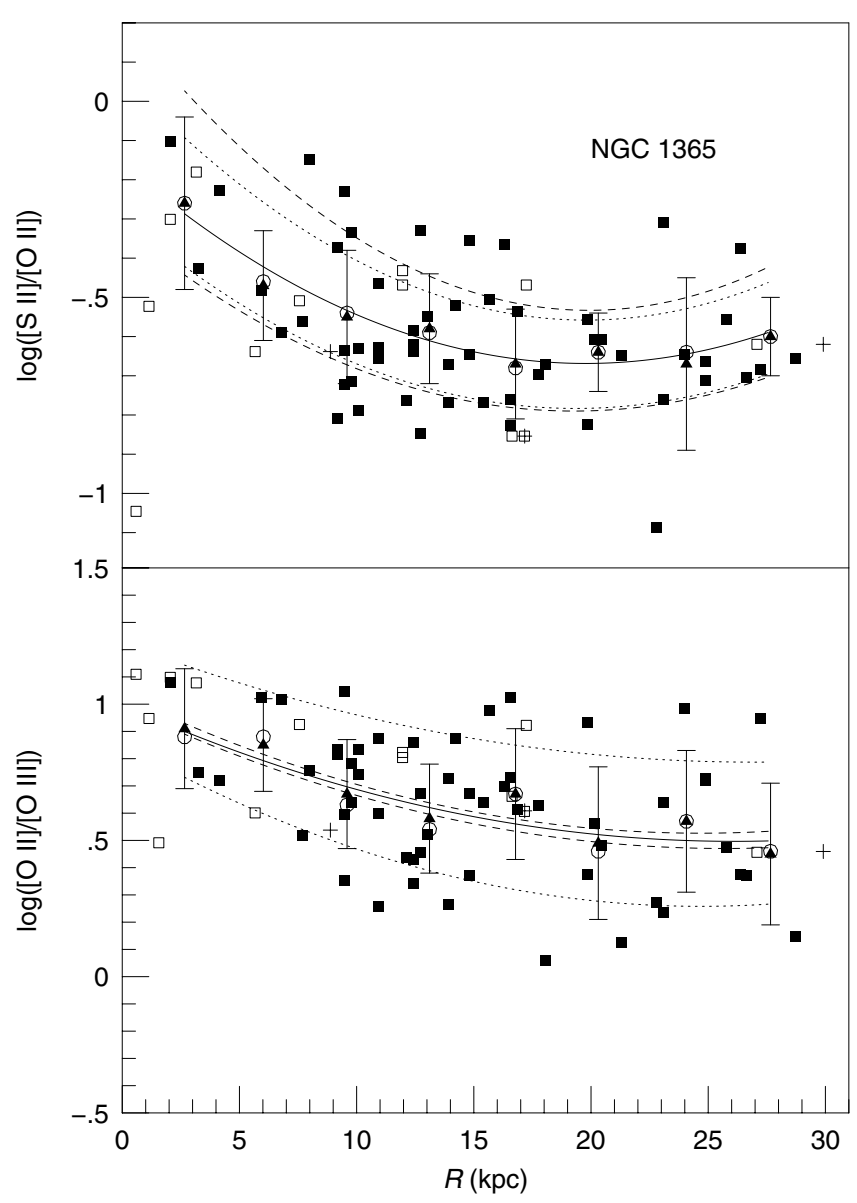

Fig. 4. $[\mathrm{OII}] /[\mathrm{O} I \mathrm{II}]$, and $[\mathrm{S} \mathrm{II}] /[\mathrm{O} \mathrm{II}]$ ratios vs. galactocentric distance $R$ for NGC 1365. The filled and open squares represent the observational data from Roy \& Walsh (1997) and from Alloin et al. (1981), respectively, while the plus signs represents the observational data from Pagel et al. (1979). The lines, triangles, open circles, and error bars are as in Fig. 1.

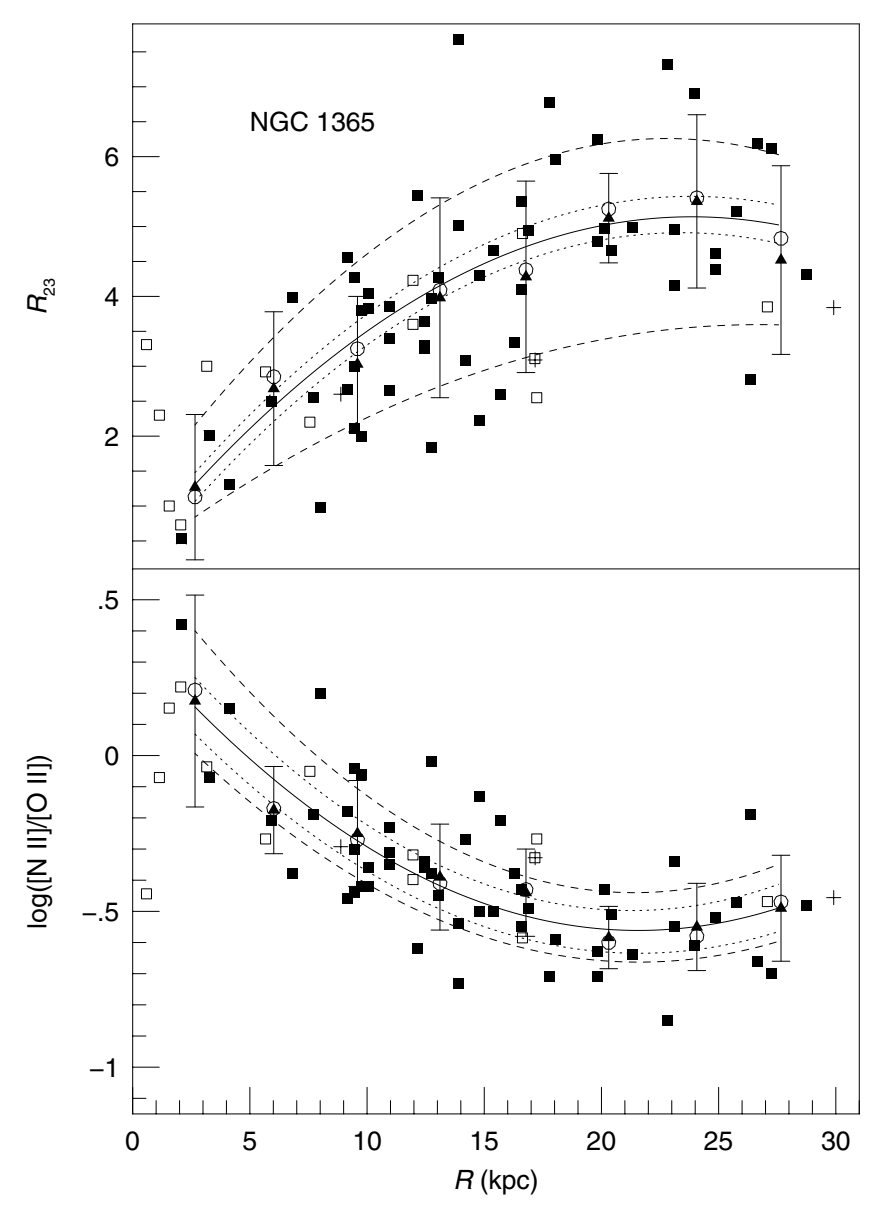

Fig. 5. Same as Fig. 4 but for $[\mathrm{N}$ II $] /[\mathrm{O}$ II $]$ and $R_{23}$. 
O. L. Dors Jr. and M. V. F. Copetti: Abundance gradients in barred galaxies, Online Material p 3

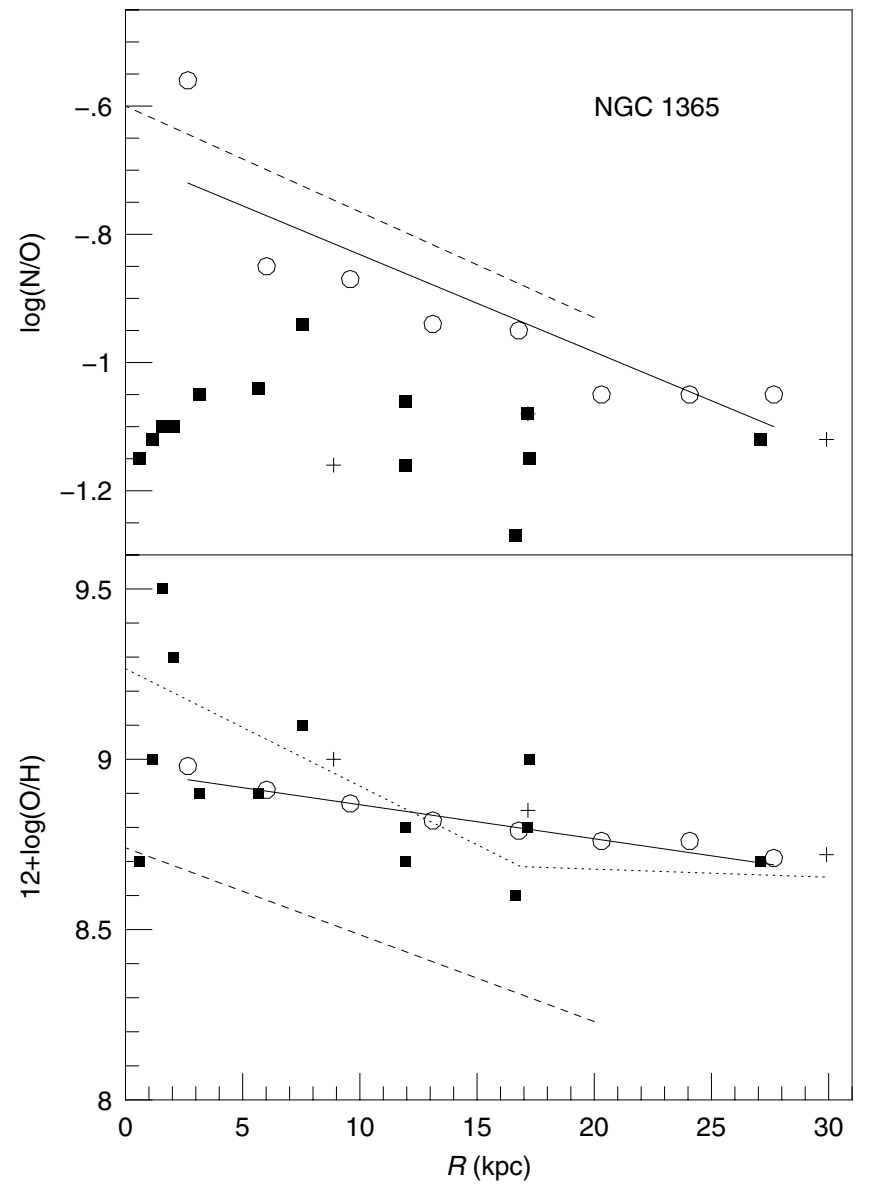

Fig. 6. Gradients of $12+\log (\mathrm{O} / \mathrm{H})$ and N/O in NGC 1365 . The solid lines are linear regressions of our best models represented by open circles. The plus signs and squares represent empirical estimates by Pagel et al. (1979) and Alloin et al. (1981), respectively. The dotted and dashed lines represent the gradients obtained by Roy \& Walsh (1997) and Pilyugin et al. (2004), respectively.

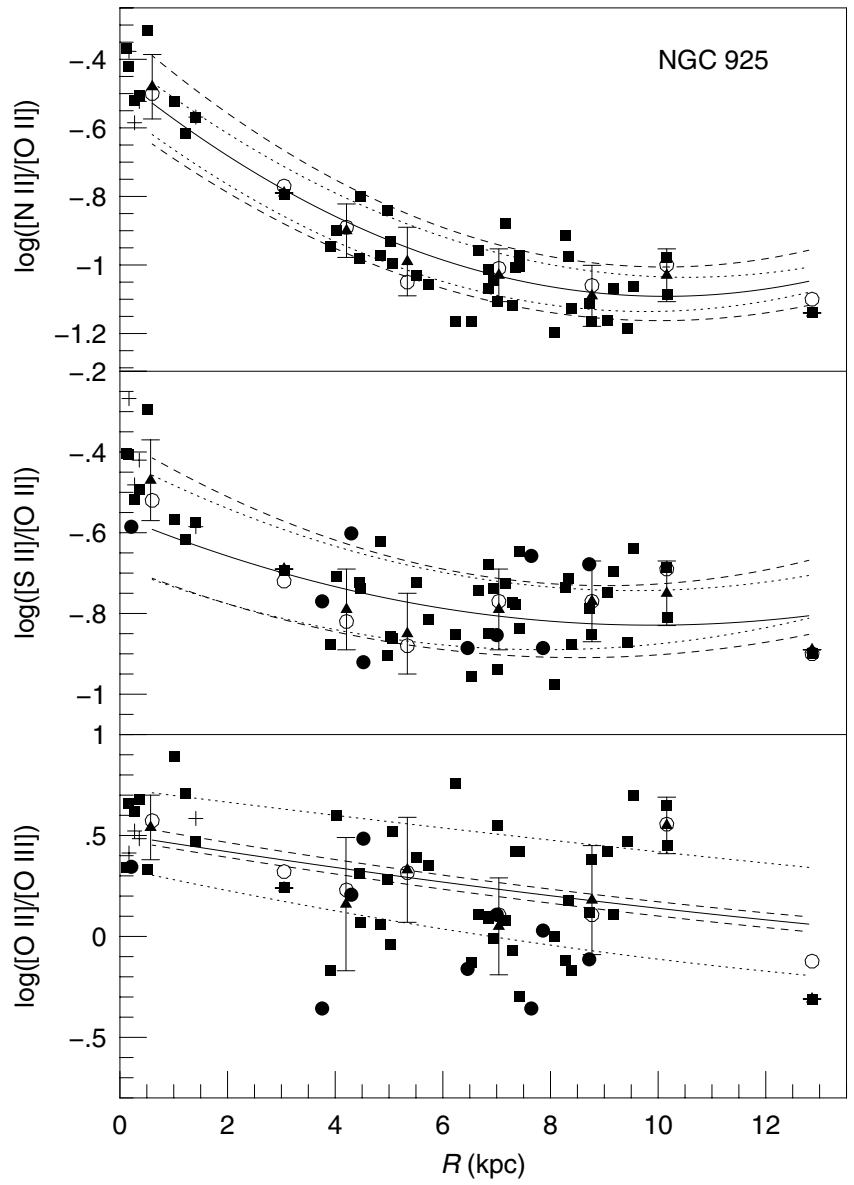

Fig. 7. [N II]/[O II], [S II]/[O II], and [O II]/[O III] ratios vs. galactocentric distance $R$ for NGC 925 . The filled squares and circles are the observational data from van Zee et al. (1998) and Zaritsky et al. (1994), respectively, while the plus signs are the ones from Castellanos et al. (2002). The lines, triangles, open circles, and error bars are as in Fig. 1. 
O. L. Dors Jr. and M. V. F. Copetti: Abundance gradients in barred galaxies, Online Material p 4

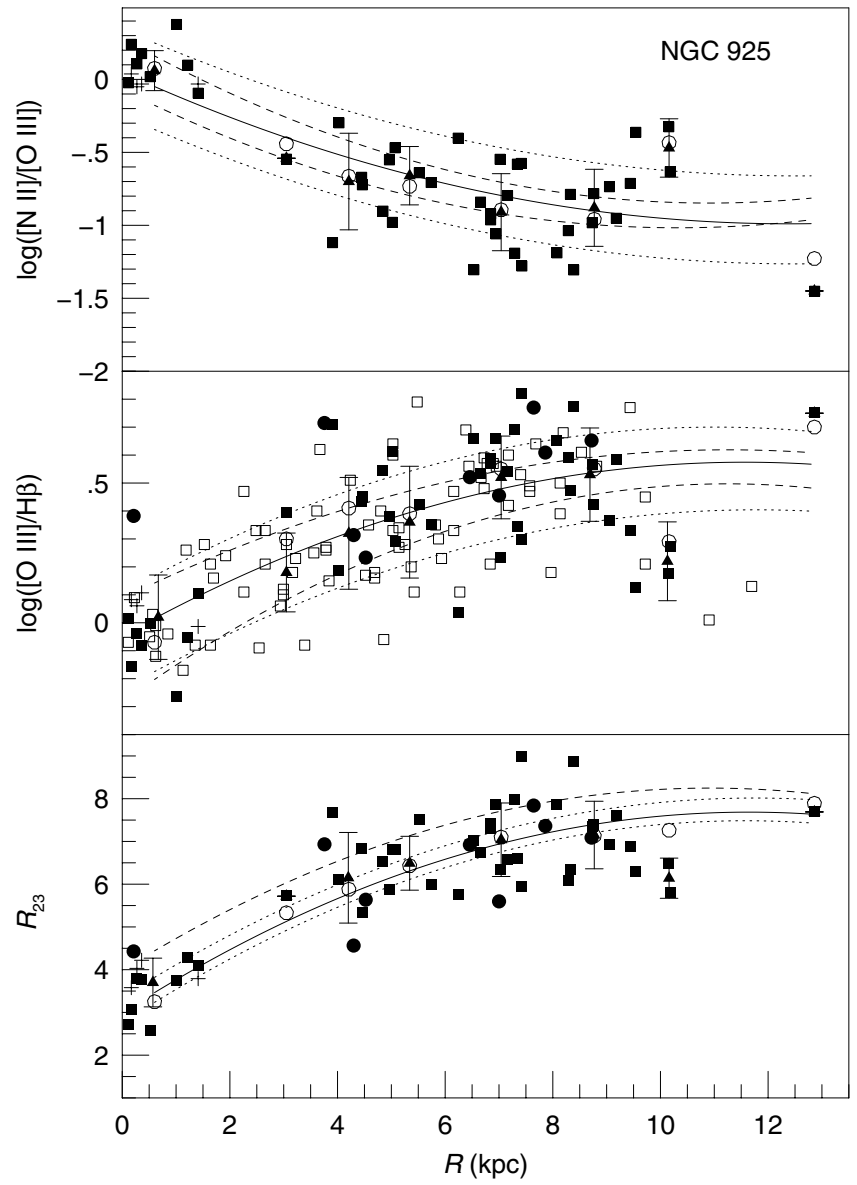

Fig. 8. Same as in Fig. 7 but for $[\mathrm{N} \mathrm{II}] /[\mathrm{O} \mathrm{III]}],[\mathrm{O} \mathrm{III}] / \mathrm{H} \beta$, and $R_{23}$. The open squares represent observational data from Martin \& Roy (1994).

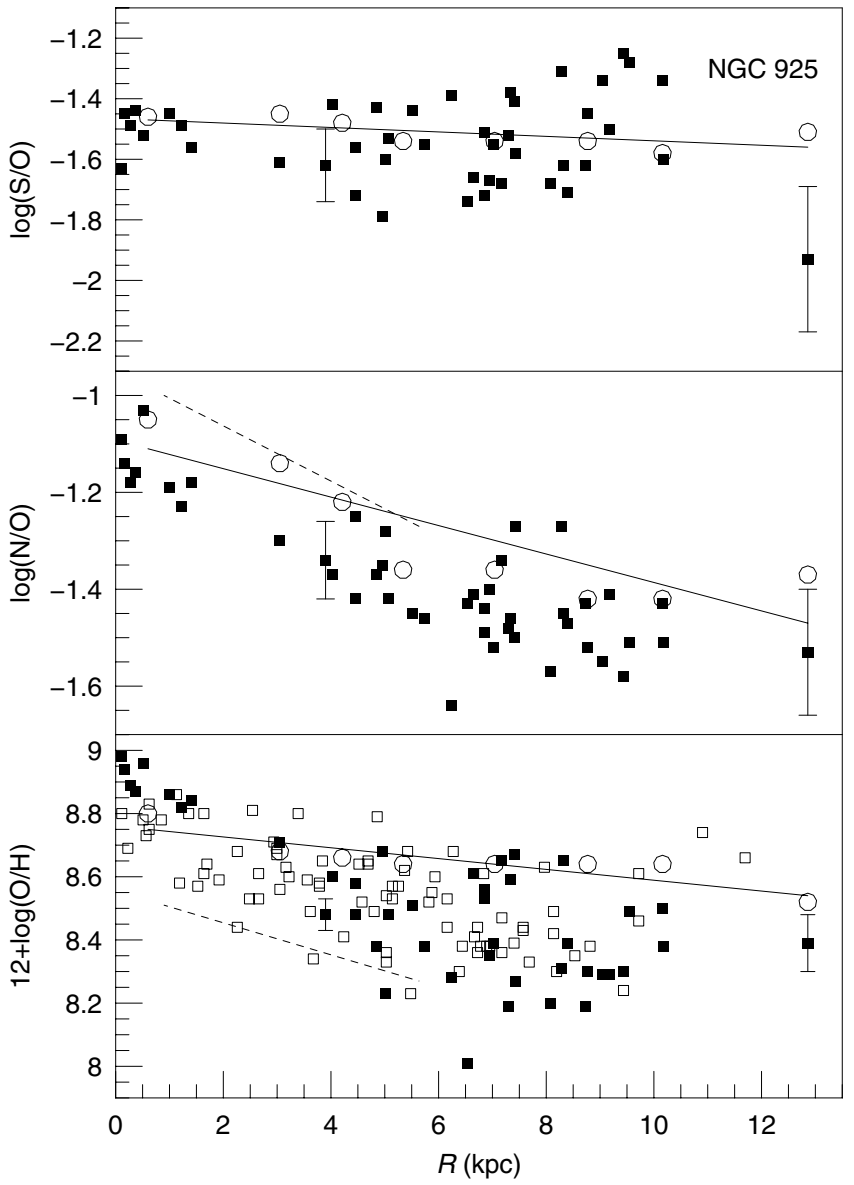

Fig. 9. Gradients of $12+\log (\mathrm{O} / \mathrm{H}), \mathrm{N} / \mathrm{O}$, and $\mathrm{S} / \mathrm{O}$ in NGC 925. The solid lines are linear regressions of our best models represented by open circles. The filled and open squares represent the abundance ratio determinations by van Zee et al. (1998) and by Martin \& Roy (1994), respectively. The points with error bars represent the direct abundance determinations by van Zee et al. (1998). The dashed lines represent abundance gradients obtained by Pilyugin et al. (2004). 
O. L. Dors Jr. and M. V. F. Copetti: Abundance gradients in barred galaxies, Online Material p 5

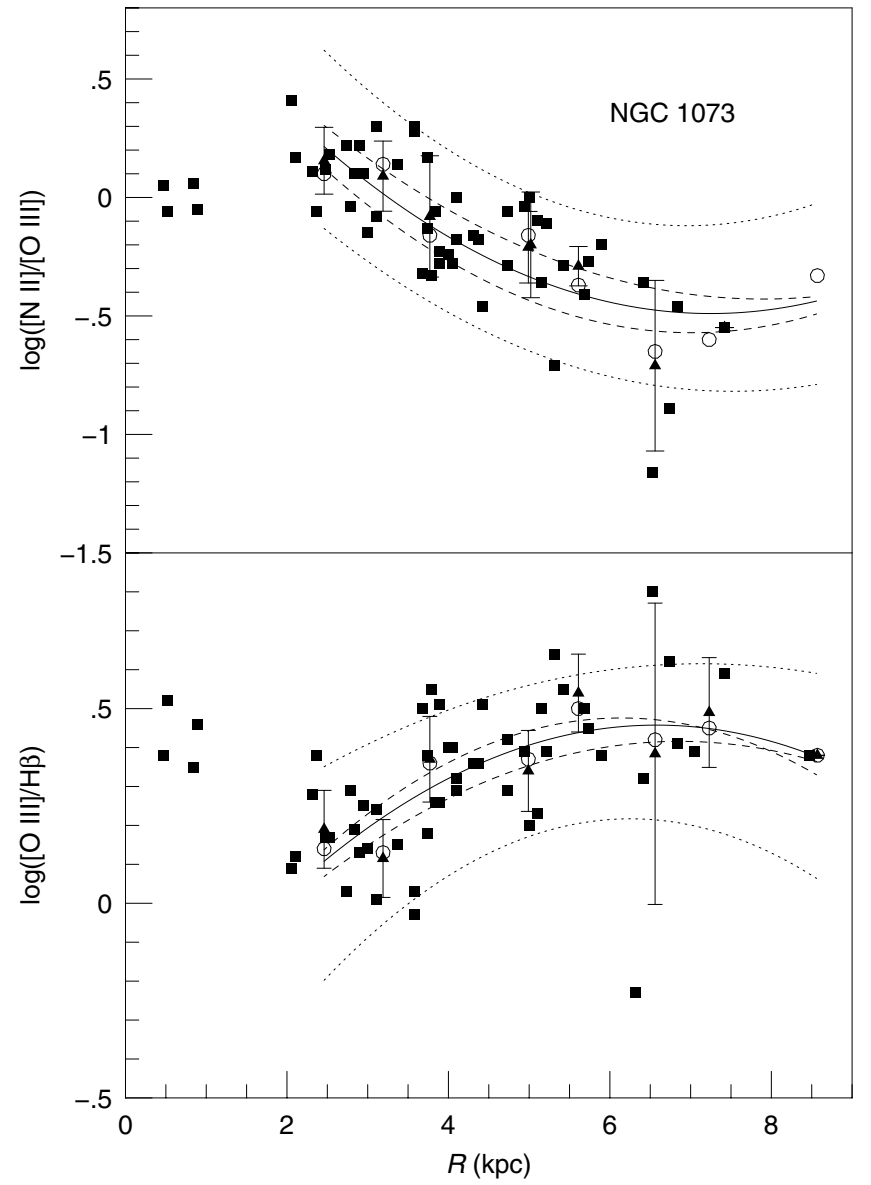

Fig. 10. $[\mathrm{N} \mathrm{II}] /[\mathrm{O} \mathrm{III}]$ and $[\mathrm{O} \mathrm{III}] / \mathrm{H} \beta$ ratios vs. galactocentric distance $R$ for NGC 1073. The filled squares represent the observational data from Martin \& Roy (1994). The lines, triangles, open circles, and error bars are as in Fig. 1.

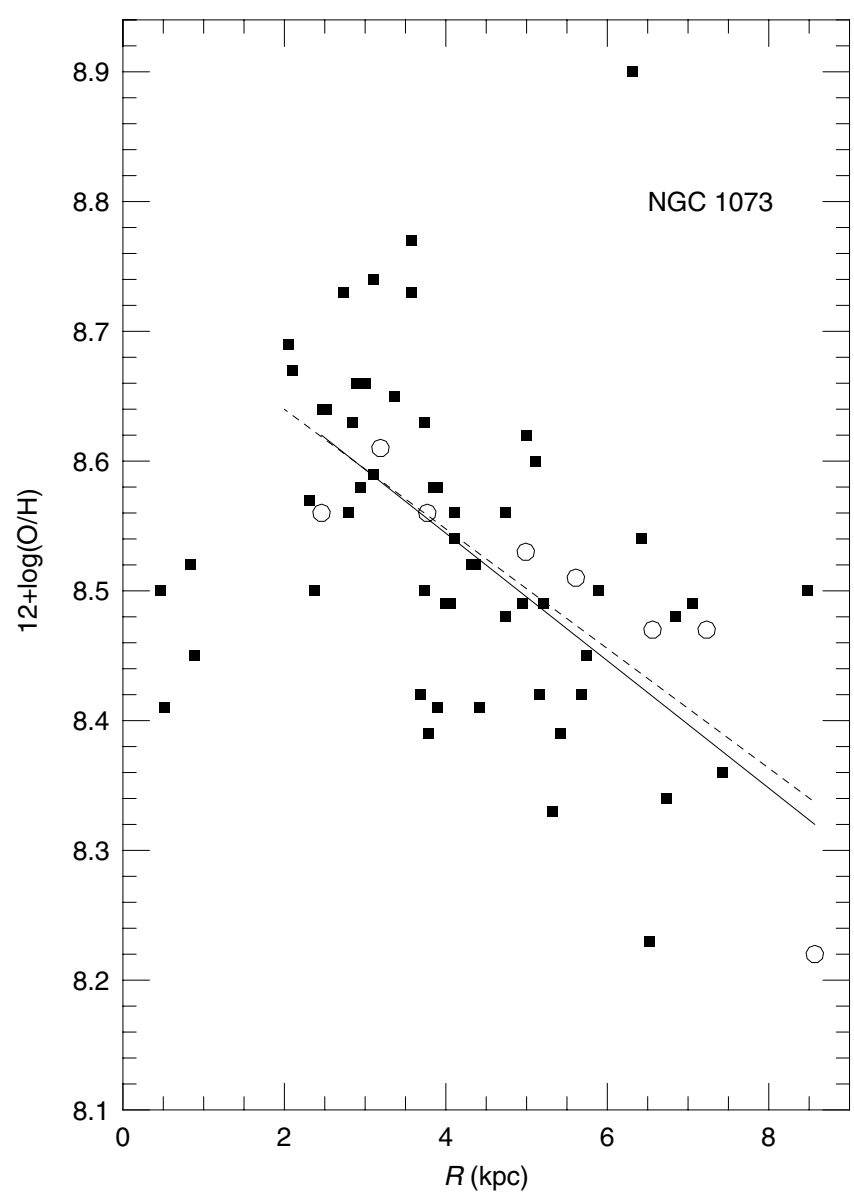

Fig. 11. Gradient of $12+\log (\mathrm{O} / \mathrm{H})$ in NGC 1073. Squares represent the O/H determinations by Martin \& Roy (1994) and the dashed line represents a linear fit for these data. The solid lines are linear regressions of our best models represented by open circles. 
O. L. Dors Jr. and M. V. F. Copetti: Abundance gradients in barred galaxies, Online Material p 6

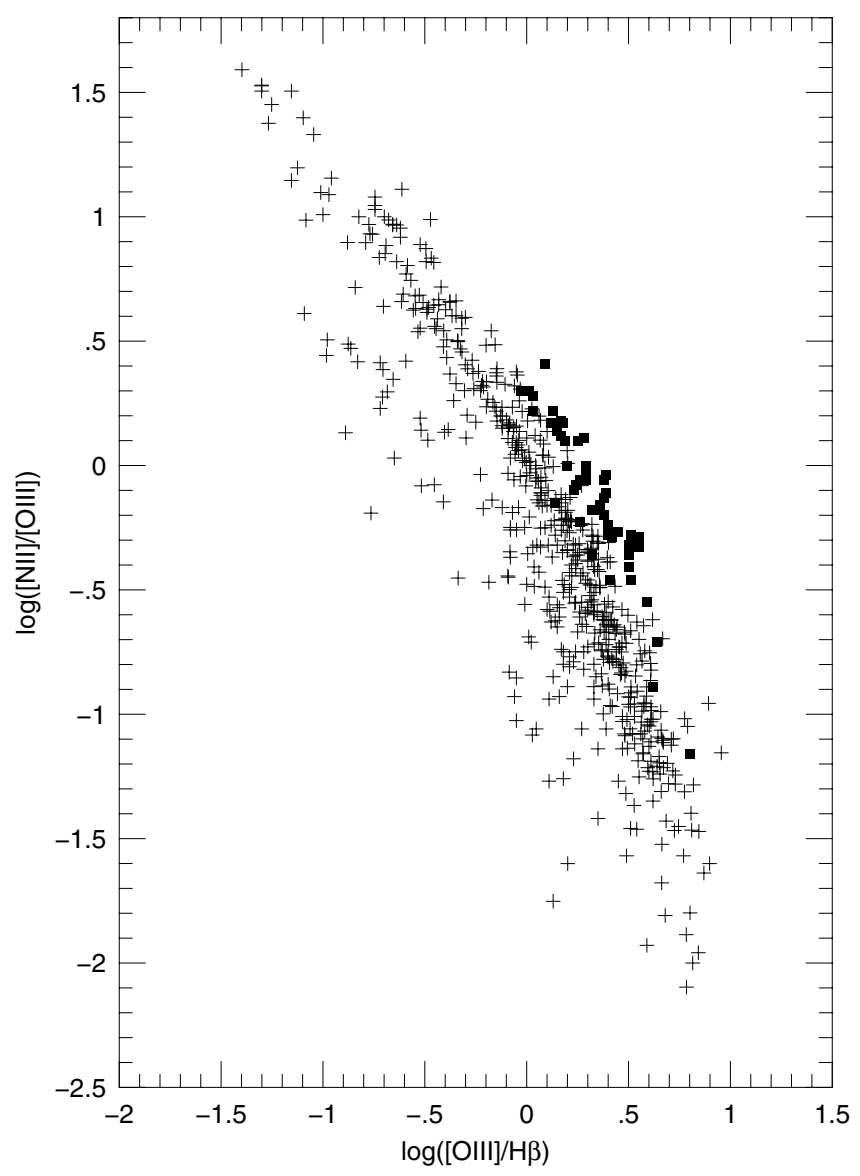

Fig. 12. $[\mathrm{N} \mathrm{II}] /[\mathrm{O} \mathrm{III}]$ vs. $[\mathrm{O} \mathrm{III}] / \mathrm{H} \beta$. The squares represent the observational data by Martin \& Roy (1994) for H II regions in NGC 1073, while the plus signs represent the ones compiled from the literature (see text) for H II regions in 34 spiral galaxies. 